Species diversity in the Antrodia crassa group (Polyporales, Basidiomycota)

\author{
Spirin, Viacheslav
}

2015-12

Spirin , V , Runnel , K, Vlasák , J , Miettinen , O \& Põldmaa , K 2015 , ' Species diversity in the Antrodia crassa group (Polyporales, Basidiomycota) ' , Fungal Biology , vol. 119 , no. 12 , pp. 1291-1310 . https://doi.org/10.1016/j.funbio.2015.09.008

http://hdl.handle.net/10138/159406

https://doi.org/10.1016/j.funbio.2015.09.008

Downloaded from Helda, University of Helsinki institutional repository.

This is an electronic reprint of the original article.

This reprint may differ from the original in pagination and typographic detail.

Please cite the original version. 


\section{Species diversity in the Antrodia crassa group (Polyporales, Basidiomycota)}

\section{Viacheslau SPIRIN ${ }^{a}$, Kadri RUNNEL ${ }^{b, *}$, Josef VLASÁK ${ }^{c}$, Otto MIETTINEN ${ }^{a}$, Kadri PÕLDMAA ${ }^{b}$}

a Botanical Unit (Mycology), Finnish Museum of Natural History, University of Helsinki, Unioninkatu 44, 00170 Helsinki, Finland

${ }^{\mathrm{b}}$ Department of Botany, Institute of Ecology and Earth Sciences, University of Tartu, Lai 40, EE-51015 Tartu, Estonia

${ }^{\mathrm{c} B i o l o g y}$ Centre of the Academy of Sciences of the Czech Republic, Branišouská 31, 37005 České Budějovice, Czech Republic

\section{A R T I C L E I N F O}

\section{Article history:}

Received 18 July 2015

Received in revised form

6 September 2015

Accepted 25 September 2015

Available online 9 October 2015

Corresponding Editor:

Martin I. Bidartondo

Keywords:

Host specificity

Internal transcribed spacer

Polypores

Taxonomy

Translation elongation factor

Wood-inhabiting fungi

\begin{abstract}
A B S T R A C T
Antrodia is a polyphyletic genus, comprising brown-rot polypores with annual or shortlived perennial resupinate, dimitic basidiocarps. Here we focus on species that are closely related to Antrodia crassa, and investigate their phylogeny and species delimitation using geographic, ecological, morphological and molecular data (ITS and LSU rDNA, tef1). Phylogenetic analyses distinguished four clades within the monophyletic group of eleven conifer-inhabiting species (five described herein): (1)A. crassa s. str. (boreal Eurasia), Antrodia cincta sp. nova (North America) and Antrodia cretacea sp. nova (holarctic), all three being characterized by inamyloid skeletal hyphae that dissolve quickly in $\mathrm{KOH}$ solution; (2) Antrodia ignobilis sp. nova, Antrodia sitchensis and Antrodia sordida from North America, and Antrodia piceata sp. nova (previously considered conspecific with A. sitchensis) from Eurasia, possessing amyloid skeletal hyphae; (3) Antrodia ladiana sp. nova from the southern part of the USA, Antrodia pinea from East Asia, and Antrodia ferox - so far known from subtropical North America, but here reported also from Eurasia. These three species have inamyloid hyphae and narrow basidiospores; (4) the North American Antrodia pini-cubensis, sharing similar morphological characters with A. pinea, forming a separate clade. The habitat data indicate that several species are threatened by intensive forestry.
\end{abstract}

(c) 2015 The British Mycological Society. Published by Elsevier Ltd. All rights reserved.

\section{Introduction}

The polypore genus Antrodia was introduced by Karsten (1879). Later Donk $(1960,1966)$ redefined the genus and selected Daedalea serpens Fr. as its type. Since then Antrodia has grown to one of the largest genera of polypores, including more than 50 species causing brown-rot of various trees (Kirk et al. 2008). The species in Antrodia sensu lato are characterized by resupinate to effused-reflexed basidiocarps, dimitic hyphal system with clamped generative hyphae and mostly

\footnotetext{
* Corresponding author. Tel.: +372 55981306; fax: +372 7375830.

E-mail address: kadri.runnel@ut.ee (K. Runnel).
}

http://dx.doi.org/10.1016/j.funbio.2015.09.008

1878-6146/@ 2015 The British Mycological Society. Published by Elsevier Ltd. All rights reserved. 
colourless skeletal hyphae, and smooth, thin walled and inamyloid basidiospores (Ryvarden \& Gilbertson 1993). Recent phylogenetic analyses based on molecular data have proven the genus to be polyphyletic as the 'core Antrodia' group comprises, besides Antrodia species that cluster in several strongly supported subsets, also species of Daedalea, Fomitopsis, and Rhodonia (Ortiz-Santana et al. 2013; Spirin et al. 2013).

Wood-inhabiting fungi in general, and the species in genus Antrodia in particular, merit attention because of their functional roles in forest ecosystems, as well as for their practical role in forest conservation. Species of this genus participate in decaying fallen dead-wood, thereby modulating the availability of resources for several other organism groups. Some Antrodia species are extremely abundant and their functional role may thus be significant in some forest systems. Other Antrodia species have become rare and threatened due to intensive forestry and deserve attention for practical use as indicators of old-growth forest habitat (e.g. Niemelä 2005; Kotiranta et al. 2010). However, their value as indicator species appears to be obscure (Runnel et al. 2014) due to the scarcity of comparative ecological and taxonomic studies.

Antrodia crassa (P. Karst.) Ryvarden is a perennial polypore occurring on gymnosperm hosts in the Northern Hemisphere (Gilbertson \& Ryvarden 1986; Ryvarden \& Gilbertson 1993; Núñez \& Ryvarden 2001). In taxonomical literature it has also been placed in the genus Amyloporia (Bondartsev 1953; Vampola \& Pouzar 1992; Cui \& Dai 2013), typified with Amyloporia xantha (Fr.) Bondartsev \& Singer, and appears to be closely related to the recently described Antrodia pinea (Cui \& Dai 2013). Antrodia crassa has been used as an indicator of natural conifer forests in North Europe (Trass et al. 1999; Niemelä 2005). Recent DNA analyses of European specimens, however, showed that A. crassa includes two sibling species, differing in their ecological requirements and possibly geographic range (Runnel et al. 2014). In addition, another species, growing in old-growth forests in Europe and so far called Antrodia sitchensis, was detected as closely related to A. crassa.

The aim of the present study was to evaluate the identity A. crassa, and its kin species in what we call the A. crassa group. For that we studied type specimens of A. crassa, A. pinea, A. sitchensis, Antrodia sordida, and 108 specimens identified to belong to these species as well as some morphologically similar species. After a comparison of morphological, ecological, geographic, and molecular data (from nuclear ITS, LSU, and tef1), we concluded that $A$. crassa group encompasses eleven species, including five species that are new to science. Results of this study are described and illustrated below.

\section{Materials and methods}

\section{Morphology}

Type specimens and other collections from herbaria BJFC, BPI, CFMR, H, MICH, NY, O, PRM, TAAM, TU, as well as from private herbarium of the author JV, were studied and sequenced. Herbarium acronyms are given according to Thiers (2015). Terminology used in morphological descriptions, as well as microscopic routine and the pore measuring technique follow Miettinen et al. (2006, 2012). The following abbreviations are used in the species descriptions: $\mathrm{L}$ - mean basidiospore length, $\mathrm{W}$ - mean basidiospore width, $\mathrm{Q}$ - mean length/width ratio, $\mathrm{n}$ - total number of measurements per specimens measured.

\section{DNA extraction, PCR, and sequencing}

For most of the specimens, DNA was extracted and the nuclear internal transcribed spacer (ITS) and large subunit (LSU) rDNA; and translation elongation factor 1-a (tef1) regions sequenced applying the protocols described by Tamm \& Põldmaa (2013) or Spirin et al. (2013). ITS region was amplified using the primers ITS1F (Gardes \& Bruns 1993) or ITSOF-T (Tedersoo et al. 2008) and ITS4 (White et al. 1990), and the first two domains of LSU using primers LROR and LR5 (http://biology.duke.edu/fungi/mycolab/primers.htm). The large exon of tef1 was amplified with primers tef1-983.2f (GCHYCHGGNCAYCGTGAYTTYAT) and tef1-G2r (GCDATRTGNGCRGTRTGRCARTC), obtained by modifying the original primers by Matheny et al. (2007). PCR products were sequenced at Macrogen Inc. (Amsterdam, The Netherlands) or at the Genomics laboratory of the Biology Centre, Academy of Sciences of the Czech Republic (České Budějovice, Czech Republic). For the two oldest herbarium specimens (Davidson FP-105587 and Lowe 7834), the primer combinations ITS1F/ITS2, and ITS3/ ITS4 were used. In this case PCR and sequencing followed Niskanen et al. (2009), and were performed at the Faculty of Biological and Environmental Sciences, University of Helsinki. The sequences were edited and assembled in Sequencher 5.1 (Gene Codes, Ann Arbor, MI, USA).

\section{Sequence alignment and phylogenetic analyses}

We compiled five datasets for analyses. The datasets were aligned with Mafft 7 online version (Katoh \& Toh 2008), using G-INS-I algorithm, followed by manual adjustment using GeneDoc 2.6.0.3.

(1) combined ITS-LSU dataset aiming to understand the position of species in Antrodia crassa group within Fomitopsidaceae. This dataset contained a selection of sequences of brown-rot species within Fomitopsidaceae (mostly obtained from GenBank) and species in A. crassa group (mostly produced for this study). In this dataset Antrodia albobrunnea (Romell) Ryvarden was used as the outgroup. Before analyses, 94 basepairs in the beginning of LSU region were removed from the data, because in several combined sequences this region was absent. Amyloporia nothofaginea Rajchenb. \& Gorjón was included in the dataset with sequences representing ITS region only. Gblocks (Castresana 2000) was used to select those regions of the sequences that were confidently aligned for analyses. The final dataset consisted of 45 sequences (of 23 species) with a length of 1251 basepairs.

(2) ITS dataset, containing sequences of 12 species in A. crassa group and aiming to represent all known distribution areas for each species, and older herbarium collections. This dataset was compiled, adding the ITS sequences produced for this study to the dataset published in Runnel et al. (2014); and conducting BLAST searches in available public databases (GenBank and UNITE) to retrieve additional relevant 
sequences. In this and the two following datasets, Antrodia sinuosa was used as the outgroup. The final dataset for ITS consisted of 53 sequences (28 produced for this study) with a length of 625 basepairs.

(3) tef1; (4) ITS-LSU and (5) ITS-LSU-tef1 datasets, all including a more restricted set of sequences of each of the studied species in the A. crassa group. As well as in (1), a stretch of 94 parsimony non-informative basepairs in the beginning of the LSU region were removed from the two combined multilocus datasets. The tef1 dataset consisted of 23 sequences of 598 basepairs (all produced for this study), that covered a partial large exon of tef1. The ITS-LSU dataset consisted of 33 two-locus sequences (27 LSU sequences produced for this study) with a length of 1418 basepairs. The ITS-LSU-tef1 dataset consisted of 23 three-locus sequences. Besides Antrodia pinea was included in this dataset with one combined sequence representing ITS-LSU region only. The ITS-LSU-tef1 dataset had a length of 1977 basepairs.

Data for the studied specimens and the GenBank accession numbers of their ITS, LSU, and tef1 sequences are presented in Table 1. Sequenced collections are marked by asterisk throughout the text. The final alignments for all three datasets were stored in TreeBASE (http://www.treebase.org; accession number S17962).

The three multilocus datasets were partitioned following the optimal partitioning strategy as identified in PartitionFinder 1.1.1 (Lanfear et al. 2012, 2014), using genes as initial partitions (ITS1, 5.8S, and ITS2 were considered one gene), linked branch lengths across partitions, and heuristic search algorithm (greedy algorithm). The optimal partitioning strategy identified was to analyse ITS and LSU as different partitions, and ITS and tef1 as one partition.

We conducted Maximum Likelihood (ML) and Bayesian inference (BI) phylogenetic analyses with all of the four datasets. RA $\times$ ML-HPC BlackBox 8.0.9 (Stamatakis 2014) was used for ML analyses of all datasets. The analyses were run without the invariant sites and with 1000 bootstrapping replicates. MrBayes v.3.2.1 (Ronquist et al. 2012) was used for Bayesian inference (BI) of one-gene and partitioned multilocus datasets with two parallel MCMC analyses, each consisting of seven chains, initiated from random starting trees. The analyses were run for 10000000 generations, sampling every 1000th generation. The first $25 \%$ of the trees were discarded as the burn-in; at this point, the average standard deviation of split frequencies had reached (1) 0.005 for Fomitopsidaceae ITS-LSU dataset, (2) 0.007 for ITS, (3) 0.005 for tef1, (4) 0.006 for A. crassa group ITS-LSU and (5) 0.005 for ITS-LSU-tef1 datasets. Posterior probabilities (PP) were calculated from the remaining 15002 trees in all cases. All phylogenetic analyses were run at the CIPRES Science Gateway (Miller et al. 2010).

\section{Results}

\section{Phylogenetic analyses}

ML and BI analyses generated nearly congruent topologies of the Fomitopsidaceae ITS-LSU dataset (Fig 1) as well as the
ITS (Fig 2), tef1 (App. 1) ITS-LSU (App. 2), and ITS-LSU-tef1 (Fig 3) datasets focussing on species in A. crassa group.

The analyses of combined ITS-LSU sequences of species in the Fomitopsidaceae (Fig 1) show that species in the A. crassa group form a well-supported monophyletic clade. This clade is nested within another clade that received high pp values but low BS support and comprised the type species of Amyloporia (Amyloporia xantha) and Rhodonia (Rhodonia placenta) and four other species (A. nothofaginea, Amyloporia carbonica, A. sinuosa, and Antrodia alpina).

Analyses focussing on A. crassa and its closest relatives consistently distinguished four clades within this group (Figs 2 and 3):

(A) Antrodia pini-cubensis clade, represented by a single species.

(B) Antrodia crassa clade. Three well-supported groups were distinguished in this clade. The sequences of Pinus-dwelling specimens from North-Europe, representing A. crassa, can be distinguished from the North-American Antrodia cincta sp. nov. (growing on Pinus as well), and the holarctic polyphagous A. cretacea sp. nov. The European sequences of Antrodia cretacea reveal no variation, the one originating from fruitbodies growing on Picea sp. in Minnesota, USA differs from the rest at four positions in ITS (as shown already by Runnel et al. 2014). The LSU (no tef1 sequence available) sequences from Europe and USA were, however, identical. In the analyses of the combined ITS-LSU dataset the sequence from USA clustered together with the European ones in a strongly supported group (App. 2). Taken the identical morphology of the European and the North-American specimens, we prefer to consider these as one species.

(C) Antrodia sitchensis clade. In this clade the Eurasian sequences originating from fruitbodies on Picea abies (with a few records on other conifer hosts) formed a wellsupported subclade, distinct from all North-American sequences in analyses of all four datasets (ITS: BS $=88$, $\mathrm{PP}=0.94 ;$ tef1: $\mathrm{BS}=100, \mathrm{PP}=0.91$; ITS-LSU; $\mathrm{BS}=71$, $\mathrm{PP}=0.89$; ITS-LSU-tef1: $\mathrm{BS}=89, \mathrm{PP}=0.93$ ). Based on these results, as well as the distinct geographic range, we regard these spruce-dwelling specimens, previously considered conspecific with the North-American A. sitchensis, to represent a new species - Antrodia piceata. The sequences of North-American specimens formed another subclade. Analyses of the combined ITS-LSU and ITS-LSU-tef1 datasets and the morphological studies confirm that the sequences within this subclade represent three closely related species, even though these species did not resolve in the ITS-based analyses. Namely, in the analyses of the combined data, the sequences from Arizona form a wellsupported subclade (ITS-LSU: BS $=74$ and PP $=0.71$; ITSLSU-tef1: BS $=98, \mathrm{PP}=0.99)$, described here as a new species - Antrodia ignobilis. Antrodia sordida was distinguished from $A$. sitchensis and A. ignobilis with strong support $(\mathrm{BS}=84, \mathrm{PP}=92)$ in the analyses of the three gene dataset. Analyses of the tef1 dataset (App. 1) however, did not distinguish the morphologically distinct $A$. sitchensis and $A$. ignobilis; while the analyses of the two-gene dataset did not distinguish A. sitchensis from A. sordida (App2). 
Table 1 - Data for the ITS, LSU, and tef1 sequences used in the phylogenetic analyses. Sequences produced in current study are marked with an asterisk.

\begin{tabular}{|c|c|c|c|c|c|}
\hline \multirow[t]{2}{*}{ Species } & \multirow[t]{2}{*}{ Voucher no. } & \multicolumn{3}{|c|}{ GenBank no. } & \multirow[t]{2}{*}{ Country of origin } \\
\hline & & ITS & LSU & tef1 & \\
\hline Amyloporia carbonica & CFMR FP-105585 & KC585240 & KC585062 & - & USA \\
\hline A. carbonica & DAOM F-8281 & KC585239 & KC585061 & - & Canada \\
\hline A. nothofaginea & BAFC 519794 & JF713078 & ibid & - & Argentina \\
\hline A. nothofaginea & BAFC 519796 & JF713079 & ibid & - & Argentina \\
\hline A. xantha & CFMR FP-100007 & KC585255 & KC585078 & - & USA \\
\hline A. xantha & Miettinen 12400 (X701) & JQ700269 & ibid & - & Finland \\
\hline Antrodia albobrunnea & CFMR FP-100514 & EU232215 & EU232299 & - & \\
\hline A. albobrunnea & Miettinen 13277.2 (X991) & KC595889 & ibid & - & Finland \\
\hline A. alpina & Gilbertson 6107 & KC585267 & KC585090 & - & USA \\
\hline A. alpina & CFMR FP-105523 & KC585266 & KC585089 & - & USA \\
\hline A. cincta & Gilbertson 10630 & KT711000* & - & - & USA \\
\hline A. cincta & Lowe 3393 & $\mathrm{KC585253^{ \textrm {a } }}$ & KC585075 & - & USA \\
\hline A. cincta (holotype) & Miettinen 14830 (X1711) & KT711001* & KT711028* & KT711071* & USA \\
\hline A. cincta & Vlasák 1009/12 & KT711002* & KT711029* & KT711072* & USA \\
\hline A. crassa & Helo 60707009 (X776) & KC595890 & ibid & - & Finland \\
\hline A. crassa & Junninen 6446 & KJ028071 & KT711030* & KT711069* & Finland \\
\hline A. crassa & Kinnunen 3476 & KJ028073 & KT711031* & KT711070* & Finland \\
\hline A. crassa & Spirin 6291 & KT711003* & - & - & Russia \\
\hline A. crassa & Spirin 6293 & KT711004* & - & - & Russia \\
\hline A. cretacea & Dvorak & KT711005* & - & - & Czech Republic \\
\hline A. cretacea & Gilbertson 9497 & EU232193 & EU232289 & - & USA \\
\hline A. cretacea & Lõhmus 521 & KJ028060 & - & - & Estonia \\
\hline A. cretacea & Lõhmus 946 & KJ028062 & - & - & Estonia \\
\hline A. cretacea (holotype) & Lõhmus 971 & KJ028064 & KT711032* & KT711067* & Estonia \\
\hline A. cretacea & Niemelä 8659 & KT711006* & - & - & Poland \\
\hline A. cretacea & Pennanen 368 (X1177) & KJ028059 & - & - & Finland \\
\hline A. cretacea & Spirin 2176 & KJ028065 & - & - & Russia \\
\hline A. cretacea & Spirin 4093 & KT711007* & - & - & Russia \\
\hline A. cretacea & Spirin 4285 & KT711008* & KT711033* & KT711066* & Russia \\
\hline A. cretacea & Svantesson & KT711009* & KT711034* & KT711068* & Sweden \\
\hline A. cretacea & Vlasák 1207/1 & KT711010* & - & - & Czech Republic \\
\hline A. favescens & CFMR FP-103468 & KC585268 & KC585091 & - & USA \\
\hline A. favescens & L-12838 & KC585272 & KC585095 & - & Costa Rica \\
\hline A. ferox & Klepsland 277 (X873) & KC595891 & ibid & - & Turkey \\
\hline A. ferox & Vlasák 1209/75 & KT711011* & KT711035* & KT711074* & USA \\
\hline A. heteromorpha & CFMR FP-101702 & KC585275 & KC585098 & - & USA \\
\hline A. heteromorpha & Niemelä 6348 (X686) & JQ700268 & ibid & - & Finland \\
\hline A. ignobilis (culture) & Gilbertson 11175 & KC585314 & KC585139 & KT711062* & USA \\
\hline A. ignobilis & Gilbertson 8128 & KT711012* & KT711036* & - & USA \\
\hline A. ignobilis & Lindsey 283 & KJ028049 & KT711037* & - & USA \\
\hline A. ignobilis (holotype) & Vlasák 1209/36 & KT711013* & KT711038* & KT711061* & USA \\
\hline A. ladiana (holotype) & Ryvarden 21853 & KT711014* & KT711039* & KT711077* & USA \\
\hline A. ladiana & Vlasák 1008/65 & KT711015* & KT711040* & KT711073* & USA \\
\hline A. malicola & L-13022 & KC585288 & KC585112 & - & Costa Rica \\
\hline A. malicola & BCRC 35452 & DQ013299 & AY873963 & - & Taiwan \\
\hline A. mellita & Spirin 3315 (X1333) & KC543140 & ibid & - & Russia \\
\hline A. mellita & Miettinen 2645 (X1079) & KC595897 & ibid & - & Finland \\
\hline A. piceata & Lõhmus 1989 & KJ028051 & KT711041* & KT711056* & Estonia \\
\hline A. piceata & Lõhmus 610 & KJ028053 & - & - & Estonia \\
\hline A. piceata & Lõhmus 626 & KJ028050 & - & - & Estonia \\
\hline A. piceata & Miettinen 1229 & KJ028052 & - & - & Finland \\
\hline A. piceata & Norstedt 97215 & KT711016* & KT711042* & KT711060* & France \\
\hline A. piceata & Runnel 1094 & KT711017* & KT711043* & KT711058* & Estonia \\
\hline A. piceata & Spirin 3885 & KJ028056 & KT711044* & KT711059* & Russia \\
\hline A. piceata & Spirin 4249 & KJ028055 & KT711045* & - & Russia \\
\hline A. piceata & Spirin 4384 & KJ028054 & KT711046* & KT711057* & Russia \\
\hline A. piceata & TAAM 174833 & KJ028058 & - & - & Estonia \\
\hline A. piceata (holotype) & Vlasák 1110/14 & KT711018* & KT711047* & KT711055* & Czech Republic \\
\hline A. pinea (isotype) & Cui 6522 & KC951148 & KT711048* & - & China \\
\hline A. pinea & Cui 6529 & KC951149 & - & - & China \\
\hline A. pinea & Dai 10827 & JQ837937 & - & - & China \\
\hline A. pinea & Spirin 4211 & KT711019* & - & - & Russia \\
\hline
\end{tabular}


Table 1 - (continued)

Species

Voucher no.

GenBank no.

\begin{tabular}{lll}
\hline ITS LSU & tef1
\end{tabular}

A. pini-cubensis

A. serialis

A. sitchensis

Melanoporia nigra
A. serialis

A. sinuosa

A. sinuosa

A. sitchensis

A. sitchensis

A. sitchensis

A. sordida

A. sordida

Daedalea quercina

D. quercina

Fomitopsis pinicola

F. pinicola

F. rosea

F. rosea

M. nigra

Rhodonia placenta

R. placenta

a Accessioned in Genbank as A. sordida.

Vlasák 1008/66
Boat 184
Miettinen 12401 (X732)
Miettinen 12407 (X725)
Vlasák 0509/17
CFMR FP-105587
Lowe 7834
Spirin 8782
Vlasák 0709/77
Miettinen 16954
Vlasák 0509/190
DLL 08-35
CFMR FP-103364
Miettinen 12391 (X772)
Miettinen 12439.1 (X1394)
Gilbertson 6954
Niemi 118 (X1415)
CFMR FP-90875
CFMR FP-90888
Dietz 7E
X1351

Vlasák 1008/66

Miettinen 12401 (X732)

Miettinen 12407 (X725)

Vlasák 0709/77

91 (X772)

(a)

\begin{tabular}{|c|c|c|c|}
\hline KT711020* & KT711049* & KT711076* & USA \\
\hline KC585297 & KC585121 & - & USA \\
\hline JQ700271 & ibid & - & Finland \\
\hline JQ700270 & ibid & - & Finland \\
\hline KT711021* & KT711050* & KT711075* & USA \\
\hline KT711022* & - & - & USA \\
\hline KT711023* & - & - & USA \\
\hline KT711024* & KT711051* & KT711065* & USA \\
\hline KT711025* & KT711052* & - & USA \\
\hline KT711026* & KT711053* & KT711063* & USA \\
\hline KT711027* & KT711054* & KT711064* & USA \\
\hline KC585338 & KC585163 & - & Sweden \\
\hline KC585335 & KC585160 & - & USA \\
\hline JQ700273 & ibid & - & Finland \\
\hline KC595922 & ibid & - & Bulgaria \\
\hline KC585353 & KC585181 & - & USA \\
\hline KC595923 & ibid & - & Finland \\
\hline KC585356 & KC585185 & - & USA \\
\hline KC585357 & KC585186 & - & USA \\
\hline KC585390 & KC585223 & - & USA \\
\hline KC595950 & ibid & - & Finland \\
\hline
\end{tabular}

KC595950

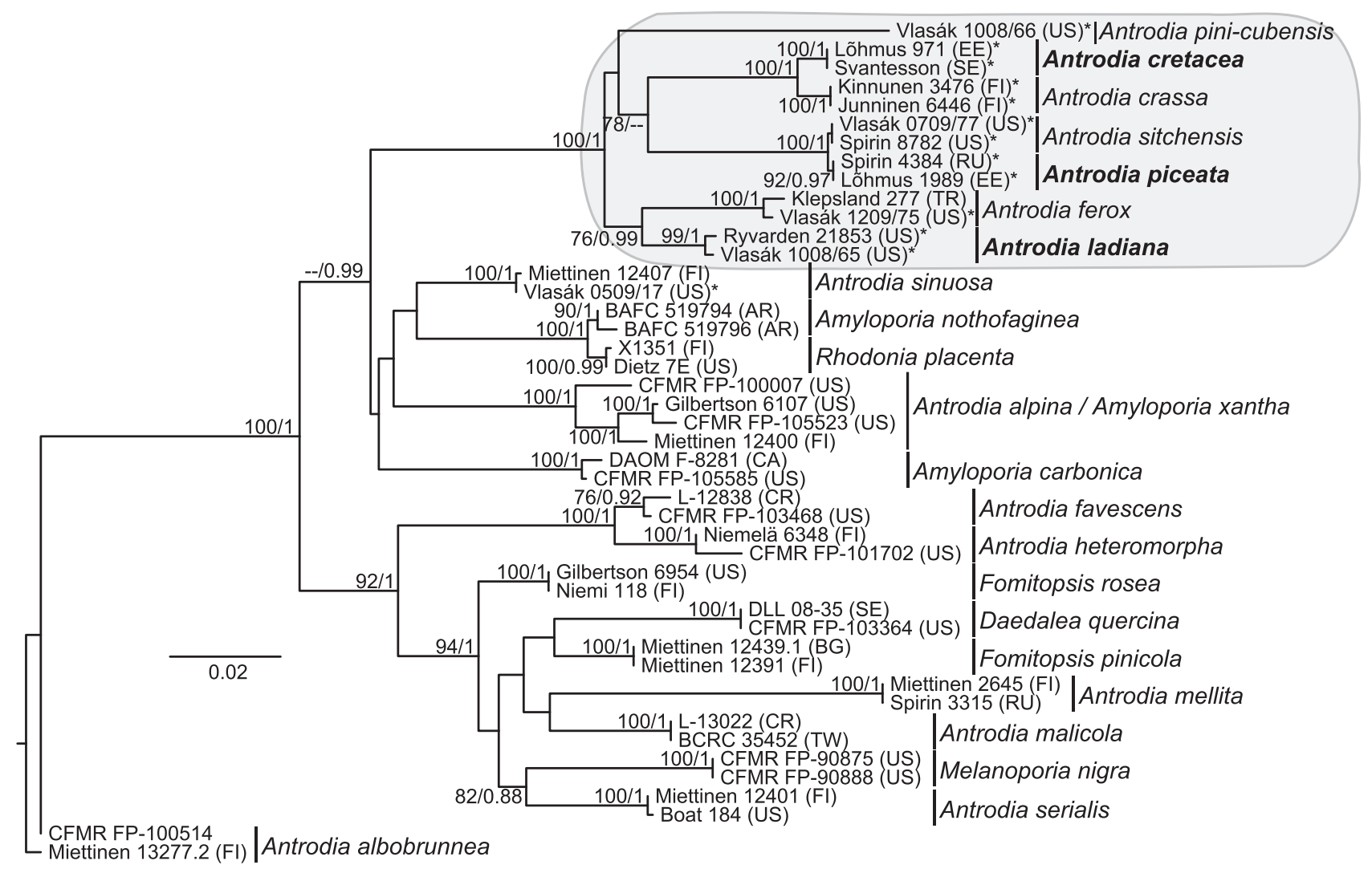

Fig 1 - Phylogenetic relationships of species in the Antrodia crassa group (coloured in grey) with other members of the Fomitopsidaceae. ML phylogram based on the ITS-LSU dataset. Numbers on nodes represent ML branch bootstrap scores ( $>70$ ) and posterior probabilities obtained from the Bayesian analysis $(>0.85)$. 


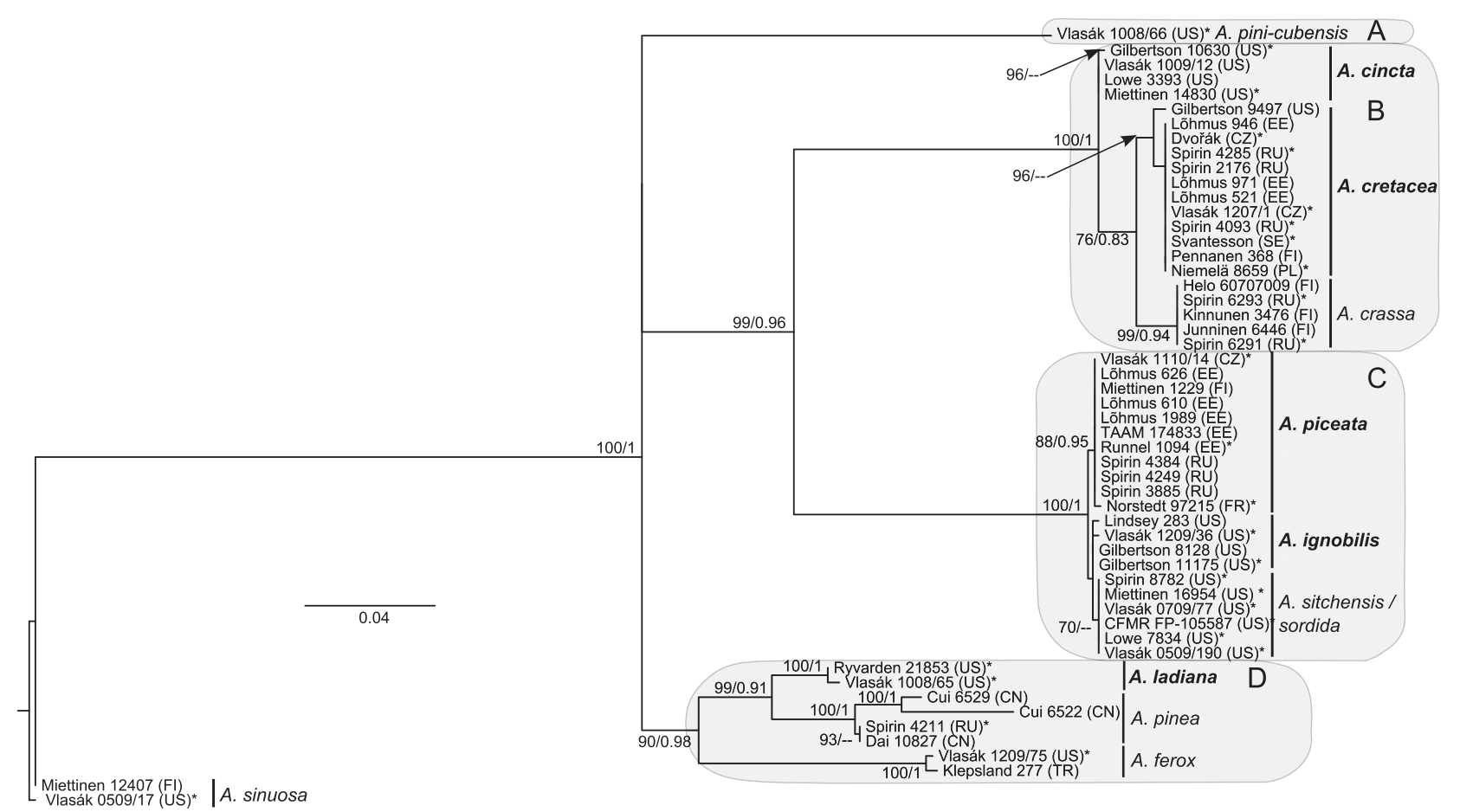

Fig 2 - Phylogenetic relationships of species in the Antrodia crassa group, inferred from the ML analysis of the ITS rDNA dataset. ML branch bootstrap $(>70)$ and posterior probability scores $(>0.85)$ are given; the sequences generated for this study are marked with an asterisk. The scale bar indicates substitutions per nucleotide position. Letters denote $A$. pini-cubensis (A), A. crassa (B), A. sitchensis (C), and A. pinea (D) clades.

(D) Antrodia pinea clade. The analyses resolved three species with maximum BS and PP scores in this clade. All of these species originate from subtropical to warm temperate zones. Antrodia ferox was so far known only from subtropical parts of North America. Besides a sequence from type locality, it is here represented by a sequence of specimen growing on Juniperus sp. in Turkey. Antrodia pinea from East Asia appears to be closely related to Antrodia ladiana, sp. nov. from the southern parts of the USA, differing in 49 characters of the ITS and nLSU rDNA (tef1 sequence for A. pinea was not available).

\section{Morphological distinction of the detected clades}

Most characteristic features for the three species in the Antrodia crassa clade are rather large but easily crumbling basidiocarps with skeletal hyphae quickly dissolving in $\mathrm{KOH}$; their basidiospores are broad, ellipsoid. Among these, A. crassa has small pores and ellipsoid basidiospores, Antrodia cretacea has wider pores and narrowly ellipsoid or thick cylindrical basidiospores, and Antrodia cincta is the small-pored and smallspored species.

Four species in the Antrodia sitchensis clade differ in having thinner, more compact basidiocarps, distinctly amyloid skeletal hyphae, which swell but do not dissolve in $\mathrm{KOH}$, and narrower, cylindrical basidiospores. The species delimitation in this group is difficult due to the minor morphological differences among species, in congruence with low interspecific variation of molecular characters studied (see above). Thus for recognition of species in this group, the geographic distribution is of major importance.

Three species in the Antrodia pinea clade are morphologically similar to A. sitchensis and its kin but their skeletal hyphae are inamyloid and subparallel in the tube trama (irregularly arranged in the A. sitchensis group). These species are distributed in warm temperate or subtropical zones. Of them, Antrodia ferox possesses fusiform to navicular basidiospores but otherwise is very similar to the closely related species. Morphological characters of Antrodia pini-cubensis resemble the members of the A. pinea clade.

\section{Species descriptions}

Antrodia cincta Spirin, Vlasák \& Miettinen, sp. nov. - Figs 4 and 5

MB 813067

Holotype. USA. Massachusetts: Worcester Co., Holden, Trout Brook, Pinus strobus, 26.IX.2011 Miettinen 14830.1* (H).

Etymology. Cinctus (Lat., adj.) - belted, delimited; referring to pronounced margin of vigorously growing basidiocarps.

Basidiocarps resupinate, effused (up to $20 \mathrm{~cm}$ in widest dimension), perennial. Margin first arachnoid to byssoid, sterile, white, in older basidiocarps indistinct, partly detaching from 


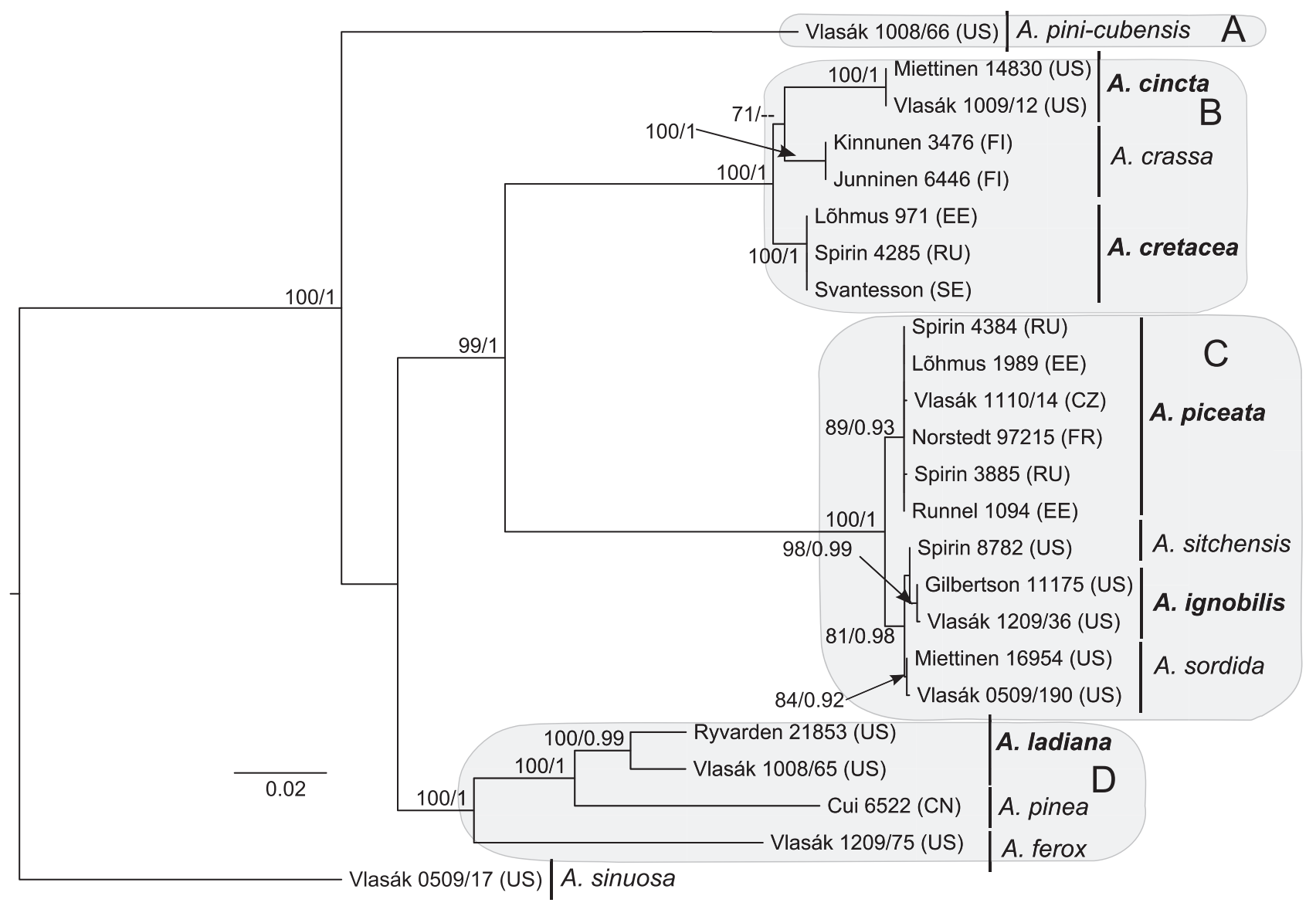

Fig 3 - Phylogenetic relationships of species in the Antrodia crassa group, inferred from ML analysis of the combined ITSLSU-tef1 dataset. ML branch bootstrap $(>70)$ and posterior probability scores $(>0.85)$ are given. The scale bar indicates substitutions per nucleotide position. Letters denote A. pini-cubensis (A); A. crassa (B), A. sitchensis (C); and A. pinea (D) clades. All tef1 sequences were generated in this study.

the substrate, dirty-brown to grayish. Pore surface white, later cream-coloured, even; pores regular, round, on sloping substrates vertically elongated, usually shallow and filled by whitish mycelium, 6-7 per $\mathrm{mm}$, dissepiments thick, opaque, uneven. Section: subiculum white, soft, and easily crumbling, up to $0.2 \mathrm{~mm}$ thick, in older basidiocarps indistinct; tubes concolorous with pore surface, cheese-like in fresh condition, fragile in herbarium specimens, indistinctly stratified, 2-5 (8) mm thick. Odour pleasant, sweet; taste weak, slightly bitter or sour.

Hyphal structure dimitic; hyphae with clamps.

Subiculum. Skeletal hyphae dominating, subsolid or solid, rarely branched, 2.7-3.8 $\mu \mathrm{m}$ in diam., inamyloid or very weakly amyloid, swelling and dissolving in $\mathrm{KOH}$, some hyphae with inflated fragments (sclerids) $4.5-6 \mu \mathrm{m}$ in diam. Generative hyphae rare, thin-walled, $2-3 \mu \mathrm{m}$ in diam.

Tubes. Skeletal hyphae dominating, irregularly arranged, thick-walled to subsolid, occasionally branched, some strongly twisted, (2.3-) 2.6-3.7 (-3.8) $\mu \mathrm{m}$ in diam. ( $\mathrm{n}=40 / 2)$, inamyloid or very weakly amyloid, distinctly swelling and dissolving in $\mathrm{KOH}$; generative hyphae thin-walled, $2.2-3.5 \mu \mathrm{m}$ in diam. Subhymenium partly distinct, consisting of thin-walled generative hyphae, up to $10 \mu \mathrm{m}$ thick. Cystidioles abundant, especially in senescent hymenium, conical, bullet- or bottleshaped, usually with blunt tips, (10.4-) 12.1-18.6 $(-20.5) \times(4.0-) 4.1-5.3(-5.4) \mu \mathrm{m}(\mathrm{n}=20 / 2)$. Basidia broadly clavate, (10.4-) 10.6-16.3 (-16.5) × (5.3-) 5.4-6.4 (-6.7) $\mu \mathrm{m}$ ( $n=20 / 2$ ). Resinous matter abundant, present as globules up to $10 \mu \mathrm{m}$ in diam.; rhomboid crystals rarely present among tramal hyphae.

Basidiospores ellipsoid to thick cylindrical (longest spores), (3.6-) 3.7-7.2 (-7.6) × (2.4-) 2.5-3.4 (-3.7) $\mu \mathrm{m}(\mathrm{n}=90 / 3)$, $\mathrm{L}=4.60, \mathrm{~W}=2.85, \mathrm{Q}=1.47-1.71$, ventral side flat, rarely slightly convex or concave, distal end evenly rounded, often with distinct oil droplets.

Discussion. Antrodia cincta is easily recognizable due to its basidiospores that are distinctly narrower and normally shorter than in Antrodia crassa and Antrodia cretacea (Table 2). Its tramal skeletal hyphae, basidia, and cystidia are more slender than in those species. The most striking macroscopic feature of A. cincta is a loose byssoid margin of vigorously growing basidiocarps. This character was mentioned by Lowe (1966) whose description of A. crassa partly covers the species in question. Antrodia cretacea, occurring in North America as 

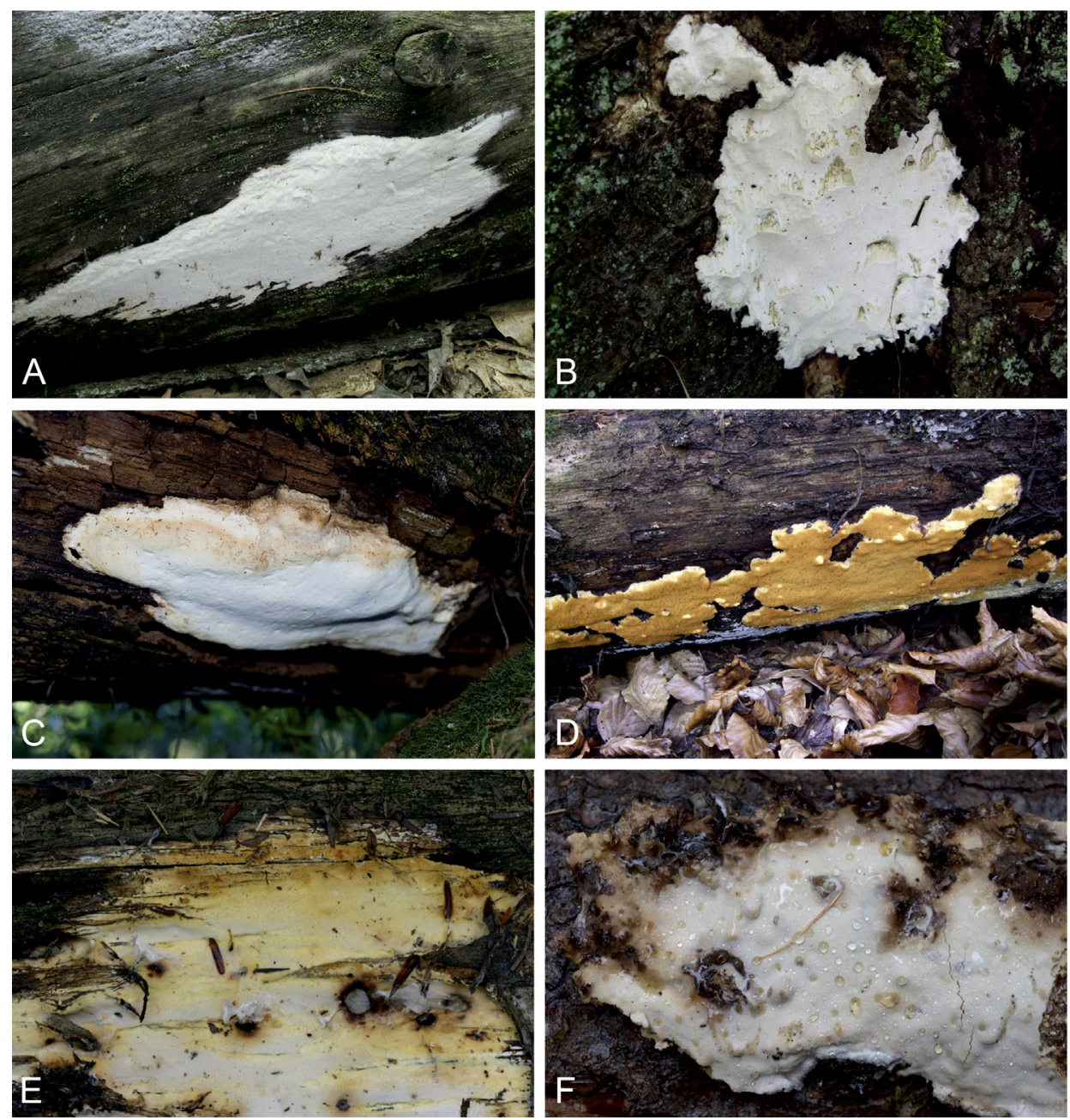

Fig 4 - Basidiocarps of the species in the Antrodia crassa group in nature: (A) A. cincta (holotype); (B) A. cretacea (Vlasák 0807/4); (C) A. crassa (photo by P. Helo; Finland. Kainuu: Sotkamo, 19.VIII.2005 Helo 1414); (D) A. piceata (J. Vlasák from the locus classicus, not collected; showing 'winter colours'); (E) A. sordida (Vlasák 0509/190); (F) A. sitchensis (Miettinen 18825). (For interpretation of the references to colour in this figure legend, the reader is referred to the web version of this article.)

well, differs in having wider pores and larger basidiospores. The latter species is not found on pine wood in North America.

Distribution and ecology. Antrodia cincta is a pine-dwelling species inhabiting large dry lying trunks. The known records of A. cincta come from both untouched and exploited forests. Here it is reported from the US North-West (from Pinus strobus) and Arizona (from Pinus ponderosa). The record of A. crassa from New Mexico (Gilbertson 1974) may refer to this species, too.

Specimens examined: USA. Arizona: Pima Co., Coronado Nat. Forest, P. ponderosa, 6.X.1970 Gilbertson 10000 (NY, O), 16.VII.1972 Gilbertson 10630* (O F-500395). Massachusetts: Worcester Co., Holden, Trout Brook, P. strobus, 26.IX.2011 Miettinen 14830.1* ( $\mathrm{H}-$ holotype, see above), 27.VII.2013 Miettinen 16625 (H). New York: Warren Co., The Pack Forest, P. strobus, 4.IX.1948 Lowe 3393* (CFMR). Pennsylvania: Adams Co., Gettysburg, Caledonia St. Park, P. strobus, 6.IX.2010 Vlasák 1009/12* (JV, H); Crawford Co., Hartstown, conifer stump, 18.XI.1929Jennings (NY).
Antrodia crassa (P. Karst.) Ryvarden, Norwegian J. Bot. 20 (1): 8, 1973 - Figs 4 and 5

$\equiv$ Physisporus crassus P. Karst., Bidr. Känned. Finlands Nat. Folk 48: 319, 1889.

Lectotype. Finland. Etelä-Häme: Tammela, Mustiala, Pinus sylvestris, 6.VI.1872 Karsten (H, selected by Lowe 1956).

Basidiocarps resupinate, effused (up to $20 \mathrm{~cm}$ in widest dimension), perennial. Margin first distinct, sterile, densely floccose, later brown, and resinous, up to $2 \mathrm{~mm}$ wide, in older specimens indistinct. Pore surface first cream-to straw-coloured, later with distinct ochraceous or brownish hues, on sloping substrates normally even; pores round, regular, on sloping substrates elongated, (5) 6-7 per mm, dissepiments thick, opaque, first even, later moderately lacerate. Section: subiculum white or near so, up to $1 \mathrm{~mm}$ thick, in older basidiocarps degenerating in chalky mass fusing with older tube layers and adjacent substrate; tubes concolorous with pore surface, cheese-like in fresh condition, relatively hard but 
Table 2 - Spore measurements of eleven species and 60 specimens in the Antrodia crassa group. All sequenced specimens are marked with an asterisk.

\begin{tabular}{|c|c|c|c|c|c|c|c|}
\hline Specimen & $\mathrm{L}^{\prime}$ & $\mathrm{L}$ & $\mathrm{W}^{\prime}$ & W & $\mathrm{Q}^{\prime}$ & Q & $\mathrm{n}$ \\
\hline A. cincta & (3.6) $3.7-7.2(7.6)$ & 4.60 & (2.4) $2.5-3.4(3.7)$ & 2.85 & (1.3) $1.4-2.2(2.6)$ & 1.61 & 90 \\
\hline holotype* & (3.6) $3.7-4.3$ (4.4) & 4.00 & $2.6-2.8(2.9)$ & 2.72 & (1.3) 1.4-1.5 (1.6) & 1.47 & 30 \\
\hline Gilbertson $10630^{*}$ & (4.0) $4.1-5.9(6.1)$ & 4.92 & (2.6) $2.7-3.4(3.7)$ & 3.00 & (1.4) $1.5-1.9(2.0)$ & 1.64 & 30 \\
\hline Vlasák 1009/12* & (3.6) $3.7-7.2$ (7.6) & 4.88 & (2.4) $2.5-3.2(3.3)$ & 2.83 & (1.3) $1.4-2.2(2.6)$ & 1.71 & 30 \\
\hline A. crassa & (4.8) $4.9-8.2(9.0)$ & 5.54 & (2.9) $3.0-3.8(4.0)$ & 3.31 & (1.4) $1.5-2.2(2.4)$ & 1.68 & 240 \\
\hline lectotype & (5.0) $5.1-8.2(9.0)$ & 5.88 & (2.9) $3.0-3.8(4.0)$ & 3.36 & (1.4) $1.6-2.2(2.4)$ & 1.74 & 30 \\
\hline Edman 845 & (5.0) 5.1-7.6 (8.1) & 5.77 & (3.0) $3.1-3.8(4.0)$ & 3.31 & (1.5) $1.6-2.0(2.1)$ & 1.74 & 30 \\
\hline Helo 60707009* & (4.9) $5.0-6.0(6.2)$ & 5.34 & (2.9) 3.0-3.7 (3.8) & 3.21 & (1.5) $1.6-1.8$ (1.9) & 1.67 & 30 \\
\hline Kinnunen $3476^{*}$ & (4.8) 4.9-6.1 (6.3) & 5.19 & (3.0) $3.1-3.5$ (3.6) & 3.25 & (1.5) $1.6-1.7$ (1.9) & 1.60 & 30 \\
\hline Lindgren 1706 & (5.0) 5.1-6.3 (6.6) & 5.64 & (3.0) $3.1-3.7(3.8)$ & 3.29 & (1.5) $1.6-1.9$ (2.0) & 1.72 & 30 \\
\hline Niemelä 6321 & (4.9) 5.0-6.1 (6.2) & 5.39 & (3.1) $3.2-3.8(3.9)$ & 3.39 & (1.4) $1.5-1.7$ (1.8) & 1.59 & 30 \\
\hline Nissinen 117 & (5.0) 5.1-6.1 (6.6) & 5.41 & (3.0) $3.1-3.5$ (3.6) & 3.25 & (1.5) 1.6-1.7 (1.8) & 1.67 & 30 \\
\hline TAAM 008597 & (5.1) 5.2-6.5 (6.6) & 5.69 & (3.0) $3.1-3.8(3.9)$ & 3.42 & (1.4) $1.5-1.8$ (1.9) & 1.67 & 30 \\
\hline A. cretacea & (4.5) $4.6-7.9(8.2)$ & 5.38 & (2.6) $2.7-4.0(4.2)$ & 3.14 & (1.3) $1.4-2.2(2.3)$ & 1.72 & 410 \\
\hline holotype* & (4.6) $4.7-6.2(7.2)$ & 5.33 & (2.7) $2.9-3.4(3.7)$ & 3.12 & $1.5-1.8(1.9)$ & 1.70 & 30 \\
\hline Lõhmus 276 & (4.5) $4.6-6.1(6.2)$ & 5.10 & (2.6) $2.7-3.3(3.7)$ & 2.94 & (1.5) $1.6-1.9$ (2.0) & 1.73 & 30 \\
\hline Dvořák 27.VIII.2006* & (4.6) $4.7-7.1(7.8)$ & 5.42 & (3.0) $3.1-3.8(4.0)$ & 3.30 & (1.4) $1.5-1.9(2.1)$ & 1.64 & 30 \\
\hline Gilbertson $9497^{*}$ & (4.5) $4.6-5.3(6.0)$ & 4.92 & (2.8) $2.9-3.3(3.5)$ & 3.06 & (1.4) $1.5-1.7$ (1.8) & 1.61 & 30 \\
\hline Niemelä 14.VIII.1969 & (4.7) $4.8-5.9(6.1)$ & 5.27 & (2.7) $2.8-3.2(3.6)$ & 2.98 & (1.5) $1.6-2.0(2.1)$ & 1.77 & 30 \\
\hline Niemelä 15.VIII.1973 & (5.0) 5.1-6.2 (6.5) & 5.59 & (3.1) $3.2-4.0(4.1)$ & 3.54 & (1.3) $1.4-1.8$ (1.9) & 1.58 & 30 \\
\hline Niemelä 5998 & (5.2) 5.3-7.8 (8.1) & 6.23 & $3.3-4.2(4.3)$ & 3.83 & (1.4) 1.5-1.8 (1.9) & 1.63 & 30 \\
\hline Pennanen 368* & (4.7) $4.8-6.3(7.2)$ & 5.47 & (2.8) 2.9-3.2 (3.9) & 3.05 & (1.5) $1.6-2.0(2.1)$ & 1.79 & 30 \\
\hline Lõhmus 521* & (4.5) $4.6-5.3(6.2)$ & 5.04 & (2.6) $2.7-3.2(3.6)$ & 2.95 & (1.5) $1.6-1.8$ (1.9) & 1.72 & 30 \\
\hline Spirin $2176^{*}$ & (4.6) $4.7-6.0(6.1)$ & 5.17 & (2.7) $2.8-3.1(3.2)$ & 2.95 & (1.6) $1.7-2.0(2.1)$ & 1.76 & 30 \\
\hline Spirin $4093^{*}$ & (4.7) $4.8-5.6$ (5.8) & 5.14 & (2.7) $2.8-3.2(3.7)$ & 2.97 & $1.6-1.9(2.0)$ & 1.74 & 30 \\
\hline Spirin $4285^{*}$ & (4.6) $4.8-5.5(5.7)$ & 5.03 & (2.6) $2.7-3.1(3.2)$ & 2.90 & $1.6-1.8(1.9)$ & 1.73 & 20 \\
\hline Tortič 81-73 & (5.1) $5.2-7.9(8.2)$ & 6.14 & (3.0) $3.1-4.0(4.2)$ & 3.39 & (1.5) $1.6-2.2(2.3)$ & 1.81 & 30 \\
\hline Vlasák 9410/6 & (4.7) 4.9-6.7 (7.2) & 5.50 & (2.7) $2.8-3.3(3.8)$ & 3.01 & (1.6) $1.7-2.0(2.1)$ & 1.83 & 30 \\
\hline A. ferox & (5.3) $5.6-9.3(10.1)$ & 6.98 & (2.5) $2.6-4.0(4.1)$ & 3.22 & (1.7) $1.8-2.7(2.8)$ & 2.18 & 60 \\
\hline Klepsland $277^{*}$ & (5.3) 5.6-7.2 (7.3) & 6.32 & (2.5) $2.6-3.3(3.4)$ & 2.81 & (1.9) $2.0-2.7(2.8)$ & 2.25 & 30 \\
\hline Vlasák 1209/75* & (6.1) 6.2-9.3 (10.1) & 7.63 & (3.3) $3.4-4.0(4.1)$ & 3.62 & (1.7) $1.8-2.5(2.6)$ & 2.11 & 30 \\
\hline A. ignobilis & (3.6) $3.7-5.3(5.7)$ & 4.31 & (1.9) $2.0-2.4(2.7)$ & 2.17 & (1.7) $1.8-2.3(2.5)$ & 1.99 & 120 \\
\hline holotype* & (3.6) $3.7-5.3(5.4)$ & 4.34 & (2.0) $2.1-2.3(2.6)$ & 2.16 & (1.7) $1.8-2.3(2.4)$ & 2.01 & 30 \\
\hline Gilbertson $8128^{*}$ & (3.7) $3.8-4.8(5.1)$ & 4.25 & (1.9) $2.0-2.4(2.7)$ & 2.12 & (1.7) $1.8-2.2(2.3)$ & 2.01 & 30 \\
\hline Gilbertson 15170 & (3.7) $3.8-5.2(5.7)$ & 4.32 & (2.0) $2.1-2.4(2.5)$ & 2.22 & (1.7) $1.8-2.2(2.5)$ & 1.95 & 30 \\
\hline Lindsey $283^{*}$ & (3.7) $3.8-5.1(5.2)$ & 4.30 & $2.0-2.4(2.7)$ & 2.17 & $1.8-2.3(2.4)$ & 1.98 & 30 \\
\hline A. ladiana & (4.4) $4.5-6.1(6.3)$ & 5.23 & (1.5) $1.6-2.1(2.2)$ & 1.81 & (2.4) $2.5-3.5(3.6)$ & 2.92 & 60 \\
\hline holotype* & (4.4) $4.5-6.1(6.3)$ & 5.25 & (1.5) $1.6-1.8$ (1.9) & 1.69 & (2.6) $2.7-3.5$ (3.6) & 3.12 & 30 \\
\hline Vlasák 1008/65* & (4.4) $4.7-5.8(6.1)$ & 5.21 & (1.7) $1.8-2.1(2.2)$ & 1.92 & (2.4) $2.5-3.1(3.2)$ & 2.72 & 30 \\
\hline A. piceata & (3.7) $3.9-6.8(7.4)$ & 4.63 & (1.6) $1.7-2.8$ & 2.10 & (1.5) $1.6-3.0(3.4)$ & 2.23 & 330 \\
\hline holotype* & (3.7) $3.9-6.1(6.2)$ & 4.49 & (2.1) $2.2-2.8$ & 2.42 & (1.5) $1.6-2.3(2.4)$ & 1.86 & 30 \\
\hline Niemelä 7300 & (3.8) $4.0-4.6(4.8)$ & 4.18 & $1.8-2.1(2.2)$ & 1.97 & (1.8) $1.9-2.4(2.5)$ & 2.14 & 30 \\
\hline Niemelä 8373 & (4.2) $4.3-7.2(7.4)$ & 4.92 & (1.8) $1.9-2.2(2.3)$ & 2.03 & (2.1) $2.2-3.1(3.2)$ & 2.42 & 30 \\
\hline Niemelä 8732 & (4.1) $4.2-6.3$ (6.6) & 4.88 & (2.0) $2.1-2.6(2.8)$ & 2.33 & (1.6) $1.7-2.5$ (2.7) & 2.11 & 30 \\
\hline Norstedt $97215^{*}$ & (4.1) $4.2-6.2(7.4)$ & 4.86 & (1.9) $2.0-2.3(2.4)$ & 2.11 & (1.8) $1.9-3.0(3.4)$ & 2.31 & 30 \\
\hline TAAM 059287 & (4.0) $4.1-5.2(5.3)$ & 4.51 & (1.6) $1.7-2.0(2.1)$ & 1.82 & (2.3) $2.4-2.8(3.0)$ & 2.49 & 30 \\
\hline TAAM $174833^{*}$ & (3.9) $4.0-5.2(5.5)$ & 4.55 & (1.6) $1.7-2.0(2.1)$ & 1.86 & (2.2) $2.3-2.6(2.7)$ & 2.45 & 30 \\
\hline Pouzar PRM 870295 & (3.8) 3.9-4.8 (5.0) & 4.24 & (2.0) $2.1-2.3(2.5)$ & 2.22 & (1.7) $1.8-2.3(2.4)$ & 1.92 & 30 \\
\hline Lõhmus 1989* & (3.8) 3.9-4.5 (4.6) & 4.25 & (1.8) $1.9-2.2(2.3)$ & 2.06 & (1.8) $1.9-2.2(2.3)$ & 2.07 & 30 \\
\hline Spirin 1401 & (4.2) $4.4-6.8$ (7.4) & 5.40 & (1.9) $2.0-2.6(2.8)$ & 2.28 & (1.8) $2.0-2.8$ (3.2) & 2.37 & 30 \\
\hline Spirin $4384^{*}$ & (4.0) $4.1-6.2(6.3)$ & 4.67 & (1.6) $1.7-2.1(2.5)$ & 1.94 & (2.0) $2.1-3.0(3.2)$ & 2.41 & 30 \\
\hline A. pinea & (4.7) $5.0-6.1(6.2)$ & 5.48 & (1.7) $1.8-2.0(2.1)$ & 1.92 & (2.3) $2.5-3.3(3.4)$ & 2.86 & 30 \\
\hline isotype* & (4.7) 5.0-6.1 (6.2) & 5.48 & (1.7) $1.8-2.0(2.1)$ & 1.92 & (2.3) $2.5-3.3(3.4)$ & 2.86 & 30 \\
\hline A. pini-cubensis & (5.1) $5.2-6.2(6.4)$ & 5.48 & (1.9) $2.0-2.2(2.3)$ & 2.09 & (2.3) $2.5-3.3(3.4)$ & 2.63 & 30 \\
\hline holotype & (5.1) 5.2-6.2 (6.4) & 5.48 & (1.9) $2.0-2.2(2.3)$ & 2.09 & (2.3) $2.5-3.3(3.4)$ & 2.63 & 30 \\
\hline A. sitchensis & (3.9) $4.1-6.8(7.6)$ & 4.80 & (1.8) $1.9-2.9$ (3.2) & 2.21 & (1.4) $1.5-2.8(3.1)$ & 2.20 & 180 \\
\hline holotype & (3.9) $4.2-6.8$ (7.6) & 4.72 & (2.2) $2.3-2.9(3.2)$ & 2.66 & (1.4) $1.5-2.4(2.5)$ & 1.79 & 30 \\
\hline DAOM 125959 & (4.4) $4.7-6.1(6.5)$ & 5.15 & (1.9) $2.0-2.3(2.4)$ & 2.08 & (2.1) $2.2-2.7$ (3.1) & 2.48 & 30 \\
\hline DAOM 125027 & (4.2) $4.3-5.5$ (5.7) & 4.86 & (1.9) $2.0-2.2(2.6)$ & 2.09 & (1.8) $1.9-2.8$ (2.9) & 2.33 & 30 \\
\hline Lowe $7834^{*}$ & (4.0) $4.1-5.0(5.2)$ & 4.43 & (1.8) $1.9-2.4(2.5)$ & 2.11 & (1.6) $1.8-2.6(2.7)$ & 2.11 & 30 \\
\hline Miettinen 18825 & (4.3) $4.5-5.7(5.8)$ & 5.03 & (1.9) $2.0-2.3$ & 2.15 & (2.0) $2.1-2.5$ (2.6) & 2.34 & 30 \\
\hline Spirin $8782^{*}$ & $4.3-5.1(5.2)$ & 4.62 & (1.9) $2.0-2.5(2.6)$ & 2.19 & (1.7) $1.8-2.4(2.6)$ & 2.12 & 30 \\
\hline
\end{tabular}


Table 2 - (continued)

\begin{tabular}{|c|c|c|c|c|c|c|c|}
\hline Specimen & $\mathrm{L}^{\prime}$ & $\mathrm{L}$ & $\mathrm{W}^{\prime}$ & $\mathrm{W}$ & $Q^{\prime}$ & $Q$ & $\mathrm{n}$ \\
\hline A. sordida & (3.6) $3.7-6.0(6.2)$ & 4.54 & (1.6) $1.7-2.2(2.3)$ & 1.85 & (1.8) $1.9-2.9(3.0)$ & 2.41 & 120 \\
\hline holotype & (4.0) $4.1-5.6(6.0)$ & 4.60 & (1.6) $1.7-1.9(2.0)$ & 1.77 & (2.4) $2.5-2.9(3.0)$ & 2.59 & 30 \\
\hline Atkinson VI.1916 & (3.9) 4.0-5.2 (5.3) & 4.43 & (1.6) $1.7-2.0(2.1)$ & 1.82 & (2.1) $2.2-2.7(2.8)$ & 2.44 & 30 \\
\hline Miettinen $16954^{*}$ & (4.1) $4.3-6.0(6.2)$ & 5.14 & $1.8-2.2(2.3)$ & 2.04 & (2.1) $2.2-2.9(3.0)$ & 2.53 & 30 \\
\hline Vlasák 0509/190* & (3.6) $3.7-4.3(4.4)$ & 3.99 & (1.6) $1.7-1.9$ (2.0) & 1.76 & (1.8) $1.9-2.4(2.5)$ & 2.07 & 30 \\
\hline
\end{tabular}

easily cut in herbarium specimens, with distinct annual layers, 2-10 $\mathrm{mm}$ thick (each layer 1-2 $\mathrm{mm}$ thick). Odour pleasant, strong, persisting in herbarium specimens; taste clearly bitter or sour.

Hyphal structure dimitic; hyphae with clamps.

Subiculum. Skeletal hyphae dominating, subsolid, 3-4 $\mu \mathrm{m}$ in diam., inamyloid, strongly swelling, and dissolving in $\mathrm{KOH}$. Generative hyphae rare, with thickened walls and oil-rich, strongly cyanophilous content, $2.5-3.5 \mu \mathrm{m}$ in diam. In older basidiocarps hyphae almost totally degenerating in amorphous crystalline mass and thus indiscernible.

Tubes. Skeletal hyphae dominating, irregularly arranged, thick-walled to subsolid, occasionally branched, some strongly twisted, (2.0-) 2.3-4.2 (-4.3) $\mu \mathrm{m}$ in diam. $(\mathrm{n}=80 / 4)$, inamyloid, strongly swelling and dissolving in $\mathrm{KOH}$; generative hyphae thin-to slightly thick-walled, some with cross (H-like) connections, 2.5-4 $\mu \mathrm{m}$ in diam. Subhymenium partly distinct, consisting of thin-walled generative hyphae, up to $15 \mu \mathrm{m}$ thick. Cystidioles abundant, especially in senescent hymenium, conical, bullet- or bottle-shaped, mostly with blunt tips, (12.3-) 12.8-22.2 (-24.3) × (4.7-) 4.8-7.6 (-8.2) $\mu \mathrm{m}$ $(\mathrm{n}=38 / 5)$. Basidia broadly clavate, (10.4-) 10.6-19.2 $(-22.6) \times(5.5-)$ 5.8-9.2 $(-10.8) \mu \mathrm{m}(\mathrm{n}=37 / 6)$. Resinous matter abundant, in young basidiocarps present as globules up to $15 \mu \mathrm{m}$ in diam., in older ones as large amorphous bodies up to $40 \mu \mathrm{m}$ in diam.; rhomboid crystals sometimes present among tramal hyphae.

Basidiospores ellipsoid to narrowly ellipsoid (longest spores), (4.8-) 4.9-8.2 (-9.0) $\times(2.9-) 3.0-3.8 \quad(-4.0) \mu \mathrm{m}$ ( $\mathrm{n}=240 / 8), \mathrm{L}=5.54, \mathrm{~W}=3.31, \mathrm{Q}=1.59-1.74$, ventral side slightly convex or flat, distal end evenly rounded.

Discussion. Antrodia crassa was described as P. crassus P. Karst. (Karsten 1889) based on several specimens collected on pine wood in Mustiala, the south-western part of Finland. The protologue represents a short formal description with a reference to Physisporus obducens (Pers.) P. Karst. (now Oxyporus obducens (Pers.) Donk) as the most similar species. The lectotype of P. crassus (selected by Lowe 1956) is a very typical, richly fertile collection.

Macroscopically, A. crassa can easily be identified due to its clearly stratified, thick, pulvinate basidiocarps with small, normally regular pores 6-7 per mm. Pore surface is usually yellowish or pale ochraceous (as if it is soaked in oil) and flat or convex; notched surface was detected only in a few very old basidiocarps more than $1 \mathrm{~cm}$ thick. The basidiospores of A. crassa are ellipsoid, constantly exceeding $3 \mu \mathrm{m}$ wide; however, their variation range mostly overlaps that of $A$. cretacea (see below).

Distribution and ecology. Antrodia crassa is distributed in North Europe and the boreal zone of Asia. It grows on tough lying trunks of P. sylvestris and Pinus koraiensis in dead-wood rich mature to old pine forests (Junninen 2009). Within such habitat this species is not very rare, although uncommon. The similarly looking A. cretacea is a more southern species with wider host range.

Specimens examined. Finland. Etelä-Häme: Tammela, Mustiala, P. sylvestris, 6.VI.1872 Karsten (H, lectotype), IX.1878, IX.1886 Karsten (H). Pohjois-Karjala: Lieksa, ResvasuoKitkasuo, P. sylvestris, 26.IX.2005 Kinnunen 3476 (H 6029178), Patvinsuo Nat. Park, P. sylvestris, 12.X.2005 Junninen 6444, 6446* (H 6029176, 6029177), Ruunaa, P. sylvestris, 7.IX.1999 Vehmaa 2428 (H); Valtimo, Salmijärvi, P. sylvestris, 26.IX.2005 Kinnunen 3476* (H 6029179). Kainuu: Suomussalmi, Pahamaailma, P. sylvestris, 15.X.2006 Helo 61015005 (H 6029180). Koillismaa: Kuusamo, Oulanka Nat. Park, P. sylvestris, 13.V.2008 Junninen 7449 (H 6029185); Salla, Kaunisharju, P. sylvestris, 6.IX.2005 Kinnunen $3348(\mathrm{H})$; Taivalkoski, Jokijärvi, P. sylvestris, 15.VIII.1994 Lindgren 1706 (H), Kylmäluoma, P. sylvestris, 7.VII.2006 Helo 60707009* (H 6029183), Metsäkylä, P. sylvestris, 16.VIII.1994 Nissinen $117(\mathrm{H})$. Perä-Pohjanmaa: Rovaniemi, Tervajuppo, P. sylvestris, 23.V.2008 Tahvonen (H 6029182). Sompion-Lappi: Sodankylä, Rautavaara, P. sylvestris, 28.VIII.2002 Hiltunen 02RH11 (H); Pekosenniemi, Luosto, P. sylvestris, 20.VIII.1998 Niemelä 6321 (H). Russia. Gornyi Altai: Altai Nat. Res., Kyga, P. sylvestris, 28.VIII.1959 Parmasto (TAAM 008597, 008011). Krasnoyarsk Reg.: Stolby Nat. Res., P. sylvestris, 12.VIII.1980 Parmasto (TAAM 102875). Primorie Reg.: Krasnoarmeiskii Dist., Melnichnoe, Pinus koraiensis, 23.VIII.2013 Spirin 6291*, 6293* (H); Ternei Dist., Maisa, P. koraiensis, 23.IX.1979, 16.IX.1990 Parmasto (TAAM 101719, 151116). Sweden. Hälsingland: Ljusdal, Sundsberget, ?P. sylvestris, 10.IX.1997 Edman 845 (H).

Antrodia cretacea Runnel, Spirin \& Lõhmus, sp. nov. Figs 4 and 5

\section{MB 813068}

Holotype. Estonia. Pärnumaa: Kanaküla, Picea abies, 8.IX.2006 Lõhmus 971* (TU 121005 - holotype, H - isotype).

Etymology. Cretaceus (Lat., adj.) - chalky; referring to the basidiocarp consistence.

Basidiocarps resupinate, widely effused (up to $40 \mathrm{~cm}$ in widest dimension), perennial. Margin first distinct, whitish to cream-coloured, sterile, densely floccose and partly detaching from the substrate, later usually brownish or grayish, degenerating in crumbling mass, up to $2 \mathrm{~mm}$ wide, in senescent basidiocarps sometimes up to $5 \mathrm{~mm}$ or even more. Pore surface first whitish to cream-coloured, later yellowishochraceous or brownish, on sloping substrates certainly stepwise-like; pores roundish to angular, on sloping substrates vertically elongated, 4-6 (7) per mm, dissepiments 
thick, opaque, first wavy, later moderately lacerate. Section: subiculum white or near so, easily crumbling, up to $1 \mathrm{~mm}$ thick, in older basidiocarps degenerating in chalky mass fusing with older tube layers and underlying substrate; tubes concolorous with pore surface or slightly paler, cheese-like in fresh condition, soft, and easily cut in herbarium specimens, with indistinct annual layers, 2-8 (10) mm thick. Odour pleasant, faint, or absent; taste slightly bitter or sour, in herbarium specimens usually indistinct.

Hyphal structure dimitic; hyphae with clamps.

Subiculum. Skeletal hyphae dominating, with capillary lumen or subsolid, 2.9-4 $\mu \mathrm{m}$ in diam., faintly amyloid (reaction indistinct), strongly swelling and dissolving in $\mathrm{KOH}$. Generative hyphae rare, with thickened walls and oil-rich, strongly cyanophilous content, 2-3.5 $\mu \mathrm{m}$ in diam. Resinous matter abundant, usually producing globose bodies up to $20 \mu \mathrm{m}$ in diam.

Tubes. Skeletal hyphae dominating, irregularly arranged, thick-walled to subsolid, occasionally branched, some strongly twisted, (2.5-) 2.6-4.1 (-4.3) $\mu \mathrm{m}$ in diam. $(\mathrm{n}=80 / 5)$, faintly amyloid (very pale violet in IKI) or inamyloid, strongly swelling and dissolving in $\mathrm{KOH}$; generative hyphae thin-to slightly thick-walled, 2.5-4 $\mu \mathrm{m}$ in diam. Subhymenium usually distinct, consisting of thin-walled generative hyphae, up to $20 \mu \mathrm{m}$ thick. Cystidioles abundant, bottle-shaped, with sharpened or blunt tips, some bifurcate, (10.8-) 12.0-21.6 $(-24.3) \times(4.1-) 4.2-6.1(-6.3) \mu \mathrm{m}(\mathrm{n}=37 / 4)$. Basidia broadly clavate, (11.3-) 13.2-20.2 (-22.6) × (4.5-) 4.7-8.1 (-8.8) $\mu \mathrm{m}$ $(\mathrm{n}=57 / 8)$. Resinous matter relatively rare in some specimens, abundant in others, in young basidiocarps present as globules up to $10 \mu \mathrm{m}$ in diam., in older ones as large amorphous bodies up to $25 \mu \mathrm{m}$ in diam. or even more.

Basidiospores narrowly ellipsoid to thick cylindrical, (4.5-) $4.6-7.9(-8.2) \times(2.6-)$ 2.7-4.0 (-4.2) $\mu \mathrm{m}(\mathrm{n}=410 / 14), \mathrm{L}=5.38$, $\mathrm{W}=3.14, \mathrm{Q}=1.58-1.83$, ventral side flat or slightly convex, rarely a bit concave, longest basidiospores slightly tapering to the distal end.

Discussion. In macroscopic terms, A. cretacea looks like an intermediate between A. crassa and Antrodia piceata/Antrodia sitchensis. The specimens we studied were mislabelled either as A. crassa or as A. sitchensis with approximately the same frequency. Antrodia crassa has denser and longer-lasting basidiocarps with smaller and more regular pores; usually, it does not produce stepwise-like growths on sloping substrates, very characteristic to A. cretacea. In older basidiocarps of the latter species the lowest tube layers and context quickly fuse into a crumbling mass, which deeply gorges the underlying substrate and thus firmly fastens them. In contrast, A. crassa mostly produces loose mycelial films filling fissures of the underlying wood; the substrate (the so-called 'kelo' - Niemelä et al. 2002) is usually so hard that the oldest basidiocarps easily detach from it.

The microscopic differences of A. cretacea from A. crassa are very subtle - basidiospores are normally thinner in A. cretacea, thick cylindrical to narrowly ellipsoid (Table 2). However, attention should be payed to the age of observed basidiocarps because senescent specimens of A. cretacea produce basidiospores which may be even wider than in A. crassa. Antrodia sitchensis in North America and A. piceata in Eurasia are macroscopically similar to A. cretacea. However, basidiospores of these species are narrower and cylindrical (Table 2, Fig 5). In addition, skeletal hyphae of these species do not change in $\mathrm{KOH}$, and possess a clear amyloid reaction. The latter character may be used for identification if the specimen at hand is sterile.

Pilát (in Kavina \& Pilát 1936-1942) listed Polyporus subfuscoflavidus Rostk. as a possible synonym of Poria crassa P. Karst. The latter species was described from Germany or, possibly, Poland (the former Prussia) as a large resupinate polypore growing on spruce. The substrate fits well with A. cretacea; however, the picture published by Rostkovius (1848) displays a largepored species with yellowish-grayish tubes. Fries's (1874) redescription of P. subfuscoflavidus refers to the oak-dwelling species found in Sweden. Unfortunately, no original collections of $P$. subfuscoflavidus exist. Romell (1926) stated an uncertain identity for this species that will be dealt with in more detail in a future study; it does not belong to the A. crassa group.

Distribution and ecology. Antrodia cretacea occurs in temperate and boreal zones of Europe, East Asia, and North America. In Europe its distribution is more southern than that of $A$. crassa, and it preferably grows on lying trunks or stumps of Picea spp. Moreover, A. cretacea is not so strict in its ecological preferences, occurring in old-growth as well as managed forests and clear-cut areas (Runnel et al. 2014).

Specimens examined. Croatia. Lika-Senja Reg.: Plitvička Jezera Nat. Park, Picea abies, 8.VIII.1973 Tortič 81-73 (H). Czech Republic. South Bohemia: Černé Údolí, P. abies, X.1994 Vlasák 9410/6 (H); Hluboká nad Vltavou, Baba, P. abies, 12.VII.2012 Vlasák 1207/1* (JV, H), Libochovka, P. abies, VIII.2007 Vlasák 0807/4 (JV). Moravia: Vývěry Punkvy, P. sylvestris, 27.VIII.2006 Duořák* (JV, H). Estonia. Pärnumaa: Kanaküla. P. abies, 6.IX.2006 Lõhmus 946* (TU 121004), 8.IX.2006 Lõhmus 971* (TU 121005 - holotype, $\mathrm{H}$ - isotype). Tartumaa: Järvselja, P. abies, X.1956 Kalmeti (TAAM 184518, 193258, 202960), 22. IX.2005 Lõhmus 276 (TU 121002), Laeva, P. abies, 1951 Pettai (TAAM 193257), Vara, P. abies, 01.X.2005 Lõhmus 521* (TU 121003). Finland. Etelä-Savo: Sulkava, Lohikoski, P. abies, 22-23.VIII.2006 Pennanen 368* (H 6012732); Poland. Podlesie Reg.: Hajnówka, Białowieża Nat. Park, P. sylvestris, 14.VIII.1969 Niemelä (H), Picea abies, 22.V.1996 Niemelä 5998 (H), 15.IX.2009 Niemelä 8659* (H). Kraków Reg.: Babia Góra Nat. Park, P. abies, 15.VIII.1973 Niemelä (H). Russia. Arkhangelsk Reg.: Plesetsk Dist., Yemtsa, P. sylvestris, 24.VIII.1965 Parmasto (TAAM 017593). Khabarovsk Reg.: Solnechnyi Dist., Gorin, Picea ajanensis, 12.VIII.2011 Spirin 4093* (H), Razlivnaya, P. ajanensis, 22.VIII.2011 Spirin 4285* (H). Leningrad Reg.: Tikhvin Dist., Choga, P. abies, 22.VII.2011 Sorokina (H). Nizhny Novgorod Reg.: Bor Dist., Kerzhenets Nat. Res., P. sylvestris, 24.IX.1998 Spirin (LE 213348); Sharanga Dist., Kilemary Nat. Res., P. abies, 18.VIII.2004 Spirin 2176* (H); Tonshaevo Dist., Kamennyi Ovrag Nat. Res., Abies sibirica, 30.V.2000 Spirin (LE 211365). Sweden. Västergötland: Vänersborg, Ovandalen, P. abies, 15.VII.2010 Suantesson* (GB). USA. Michigan: Chippewa Co., Neebish Island, Tsuga canadensis, IX.1909 Harper (NY). Minnesota: Clearwater Co., Itasca Nat. Forest, Picea sp., 1.VIII.1970 Gilbertson 9497* (O ex ARIZ - as Poria oleagina), Abies balsamea, 16.IX.1977 Ryvarden 14380 ( $\mathrm{H}$ ex O). New York: Warren Co., Warrensburg, Picea sp., 2.IX.1946 Lowe 3119 (H).

Antrodia ferox (Long \& D.V. Baxter) Gilb. \& Ryvarden, Mycotaxon 22 (2): 363, 1985 
三 Poria ferox Long \& D.V. Baxter, Pap. Michigan Acad. Sci. 25 (1): 149, 1940.

Antrodia ferox is the North American species inhabiting dead juniper wood. One specimen collected in Turkey (Klepsland 277) was found to be very similar to the North American taxon but to differ in having shorter and narrower basidiospores and slightly wider pores (Table 2). While the material is scanty, the ITS data supported its identification as the first record of A. ferox from Eurasia. A modern description of this species is presented by Gilbertson \& Ryvarden (1986).

Specimens examined. Turkey. Isparta: Eğirdir, Juniperus sp., 14.IX.2007 Klepsland 277* (O, H). USA. Arizona: Graham Co., Pinaleno Mt., Swift Trail, Juniperus scopulorum, 7.IX.2012 Vlasák 1209/75* (JV, H).

Antrodia ignobilis Spirin \& Vlasák, sp. nov. - Figs 4 and 5

MB 813070

Holotype. USA. Arizona: Pima Co., Coronado Nat. Forest, St. Catalina, P. ponderosa, 3.IX.2012 Vlasák 1209/36* (H - holotype).

Etymology. Ignobilis (Lat., adj.) - ignoble, plebeian; referring to absence of striking macroscopic features.
Basidiocarps resupinate, widely effused (up to $15 \mathrm{~cm}$ in widest dimension), perennial. Margin distinct, whitish to cream-coloured or pale ochraceous, sterile, densely floccose, in young basidiocarps partly detaching from the substrate. Pore surface first whitish to cream-coloured, later with yellowish or ochraceous hues, in oldest parts with resinous stains (as if soaked by oil); pores roundish to angular or sinuous, on sloping substrates vertically elongated and open, 6-8 per $\mathrm{mm}$, dissepiments first even, later moderately lacerate. Section: subiculum white or near so, easily crumbling, up to $1 \mathrm{~mm}$ thick, in older basidiocarps degenerating in chalky mass; tubes concolorous with pore surface or slightly paler, cheese-like in fresh condition, soft and easily cut in herbarium specimens, with distinct annual layers, (0.5) 1-5 mm thick. Odour pleasant, faint, or absent; taste slightly bitter or sour, in herbarium specimens usually indistinct.

Hyphal structure dimitic; hyphae with clamps.

Subiculum. Skeletal hyphae dominating, with capillary lumen or subsolid, (2.2-) 2.3-3.3 (-3.4) $\mu \mathrm{m}$ in diam. ( $\mathrm{n}=20 / 1)$, very faintly amyloid, swelling inwards in $\mathrm{KOH}$. Generative hyphae rare, with thickened walls and oil-rich, strongly

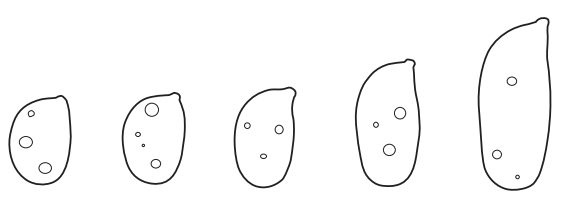

A. cincta
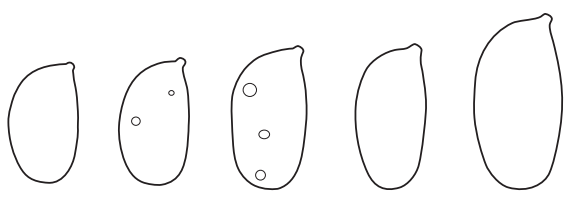

A. crassa
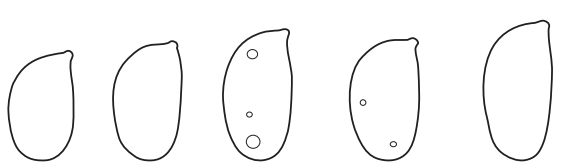

A. cretacea
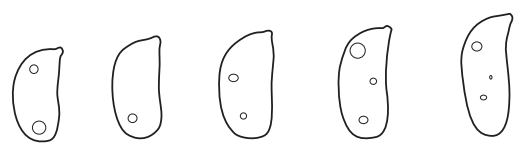

A. ignobilis

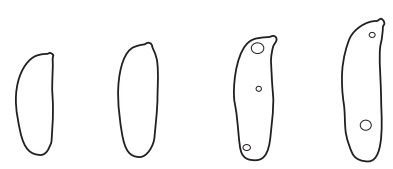

A. ladiana
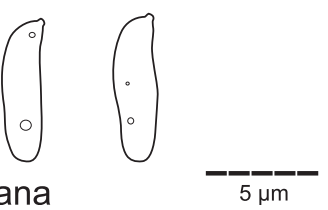
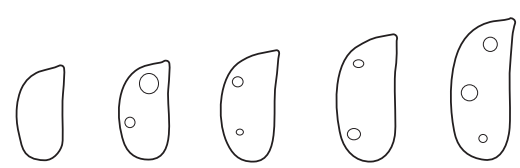

A. piceata
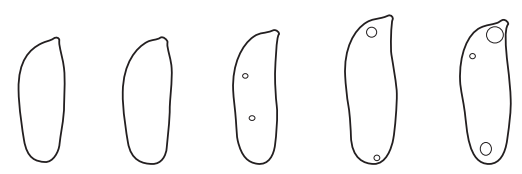

A. pini-cubensis
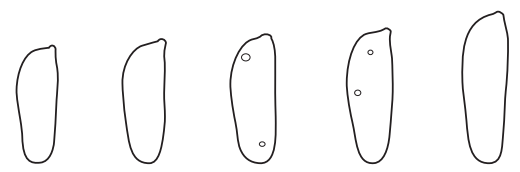

A. pinea
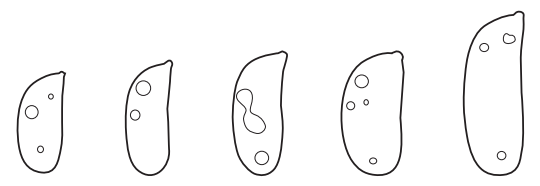

A. sitchensis
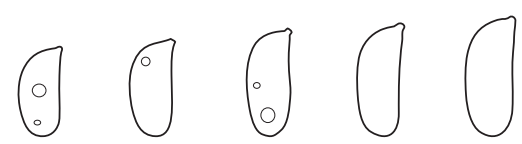

A. sordida

Fig 5 - Spore drawings of the species in the Antrodia crassa group: A. cincta (3 left - holotype, 2 right - Vlasák 1009/12), A. crassa (lectotype), A. cretacea (holotype), A. ignobilis (holotype), A. ladiana (holotype), A. piceata (holotype), A. pini-cubensis (holotype), A. pinea (holotype), A. sitchensis (3 left - Spirin 8782, 2 right - holotype), A. sordida (3 left - Vlasák 0509/190, 2 right 
cyanophilous content, 2-2.5 $\mu \mathrm{m}$ in diam. Resinous matter abundant or scarce.

Tubes. Skeletal hyphae dominating, irregularly arranged, thick-walled to subsolid, occasionally branched, some strongly twisted, (2.0-) 2.3-3.6 (-3.8) $\mu \mathrm{m}$ in diam. ( $\mathrm{n}=40 / 2)$, weakly amyloid (pale violet in IKI, in some specimens reaction indistinct), swelling inwards but not dissolving in $\mathrm{KOH}$, sparse at the tube orifices; generative hyphae thin-to slightly thickwalled, $1.5-2.5 \mu \mathrm{m}$ in diam. Subhymenium usually distinct, consisting of thin-walled generative hyphae, up to $30 \mu \mathrm{m}$ thick. Cystidioles abundant, bullet-shaped, mostly with blunt tips, (10.2-) 10.9-17.1 (-19.6) × (3.0-) 3.1-5.2 (-5.5) $\mu \mathrm{m}$ $(\mathrm{n}=25 / 2)$. Basidia clavate-pedunculate, (10.8-) 11.0-17.2 $(-17.3) \times(4.7-)$ 4.8-6.0 (-6.2) $\mu \mathrm{m}(\mathrm{n}=24 / 2)$. Resinous matter abundant, present as droplets or globules up to $15 \mu \mathrm{m}$ in diam.

Basidiospores thick cylindrical, (3.6-) 3.7-5.3 $(-5.7) \times(1.9-)$ 2.0-2.4 (-2.7) $\mu \mathrm{m}(\mathrm{n}=120 / 4), \mathrm{L}=4.31$, $\mathrm{W}=2.17, \mathrm{Q}=1.95-2.01$, ventral side slightly concave, longest spores slightly tapering to the distal end.

Discussion. A. ignobilis is morphologically similar to A. sitchensis, differing in having smaller pores and shorter spores (Tables 2 and 3). Moreover, the lowest parts of the tube dissepiments in A. ignobilis possess very scanty skeletal hyphae and therefore look almost monomitic, in contrast to the strongly dimitic dissepiment edges of A. sitchensis. Basidiocarps of A. ignobilis are often easily detaching from the substrate, possessing inrolling parts, and its senescent fruitbodies disintegrate in crumbling chalk-like mass. In addition, the distribution range of $\mathrm{A}$. ignobilis and $\mathrm{A}$. sitchensis are different.

Distribution and ecology. Antrodia ignobilis is found in Arizona and New Mexico (USA); it inhabits dry lying trunks of P. ponderosa and Pinus strobiformis. Previous records of A. sitchensis from these states (Gilbertson 1974) refer to A. ignobilis.

Specimens examined. USA. Arizona: Pima Co., Coronado Nat. Forest, P. ponderosa, 31.X.1969 Gilbertson 9330 (O), 3.IX.2012 Vlasák 1209/36* (H - holotype, JV - isotype), Pinus strobiformis, 11.VIII.1972 Gilbertson 10686 (O); Coconino Co., Coconino Nat. Forest, P. ponderosa, 21.VIII.1973 Lindsey 283* (H ex O F-910108), 22.VIII.1984 Gilbertson 15170 (CFMR). New Mexico: Otero Co., Lincoln Nat. Forest., P. ponderosa, 23.VIII.1968 Gilbertson 8128* (NY, MICH).

\section{Antrodia ladiana Spirin \& Runnel, sp. nov. - Fig 5}

\section{MB 813072}

Holotype. USA. California: Tulare Co., Sequoia Nat. Park, Pinus sabiniana, 20.III.1984 Ryvarden 21853* (O F-910054 - holotype, $\mathrm{H}-$ isotype).

Etymology. Ladianos (Greek, adj.) - greasy; referring to numerous droplets exuding in microscopic mountants.

Basidiocarps resupinate, up to $15 \mathrm{~cm}$ in widest dimension, perennial. Margin distinct, whitish to cream-coloured, sterile, densely floccose, firmly attached. Pore surface cream-coloured, later with ochraceous or light brownish hues (as if soaked by oil); pores angular, variable and of irregular size, 4-6 (7) per $\mathrm{mm}$, dissepiments thin, semitransluscent, first even, later minutely serrate. Section: subiculum white, densely floccose, up to $0.1 \mathrm{~mm}$ thick; tubes concolorous with pore surface, soft leathery and easily cut in herbarium specimens, no distinct annual layers, 0.3-1.5 mm thick. Odour absent; taste indistinct.
Hyphal structure dimitic; hyphae with clamps.

Subiculum. Skeletal hyphae dominating, irregularly arranged, with capillary lumen or subsolid, 2-3 $\mu \mathrm{m}$ in diam., inamyloid, slightly swelling in $\mathrm{KOH}$. Generative hyphae rare, with thickened walls, $2.5-3.5 \mu \mathrm{m}$ in diam. Resinous matter absent.

Tubes. Skeletal hyphae dominating, subparallel, thickwalled to subsolid, 2.0-4.1 $(-4.5) \mu \mathrm{m}$ in diam. ( $\mathrm{n}=40 / 2)$, inamyloid, slightly swelling in $\mathrm{KOH}$; generative hyphae thin-to slightly thick-walled, 2.2-3.2 (3.5) $\mu \mathrm{m}$ in diam. Subhymenium distinct in young basidiocarps, 15-20 $\mu \mathrm{m}$ thick, composed by delicate easily collapsing generative hyphae. Cystidioles abundant, bullet-shaped, with blunt tips, rarely tapering, (8.8-) 9.4-13.1 (-13.2) × (3.8-) 3.9-4.6 (-5.4) $\mu \mathrm{m}(\mathrm{n}=30 / 2)$. Basidia clavate, (8.3-) 9.2-13.1 (-15.4) × (4.1-) 4.3-5.2 (-5.3) $\mu \mathrm{m}(\mathrm{n}=30 / 2)$. Resinous matter absent, crystals occasionally present, rhomboid or polygonal, 5-15 $\mu \mathrm{m}$ wide.

Basidiospores cylindrical, (4.4-) 4.5-6.1 (-6.3) × (1.5-) 1.6-2.1 (-2.2) $\mu \mathrm{m} \quad(\mathrm{n}=60 / 2), \quad \mathrm{L}=5.23, \mathrm{~W}=1.81$, $\mathrm{Q}=2.72-3.12$, ventral side flat, rarely slightly concave, longest spores slightly tapering to the distal end.

Discussion. According to morphological and DNA data, A. ladiana is closely related to A. ferox and A. pinea from East Asia. Antrodia ferox differs in having peculiar basidiospores and it inhabits juniper wood. Antrodia pinea has smaller pores (6-8 per $\mathrm{mm}$ ) and large rhomboid crystals among hyphal tissues; it is distributed in East Asia. Antrodia pini-cubensis from the Caribbean is morphologically similar to A. ladiana as well, although it is not closely related. It differs in having more deeply coloured, bright ochraceous basidiocarps and a presence of abundant oily droplets in microscopic mounts. Microscopically, it is almost identical to A. ladiana except slightly wider cystidioles and basidia. A good hint for recognizing these species is the shape of pores which show a highly variable diameter in A. ladiana but more or less regular and in general smaller in A. pini-cubensis.

Distribution and ecology. So far the species is known only from two localities in warm temperate to subtropical regions in southwestern and souteastern USA, growing on lying trunks of Pinus spp. The specimen from Florida was collected from a charred lying trunk of Pinus elliottii, on which it was growing side by side with A. pini-cubensis.

Specimens examined. USA. California: Tulare Co., Sequoia Nat. Park, P. sabiniana, 20.III.1984 Ryvarden 21853* (O F-910054 - holotype, $\mathrm{H}$ - isotype). Florida: Collier Co., Everglades Nat. Park, P. elliottii, 29.VIII.2010 Vlasák 1008/65* (JV, H).

\section{Antrodia piceata Runnel, Spirin \& Vlasák, sp. nov. - Figs 4 and 5}

\section{MB 813073}

Holotype. Czech Republic. South Bohemia Reg.: Boubín Nat. Res., Picea abies, 29.X.2011 Vlasák 1110/14* (H - holotype).

Etymology. Piceatus (Lat., adj.) - resinous; referring to resinous-brown margin of old basidiocarps.

Basidiocarps resupinate, large, covering several decimetres, perennial. Margin distinct, first whitish to cream-coloured or pale ochraceous, sterile, leathery, firmly attached, in older basidiocarps becoming resinous, dark brown to almost black, up to $3 \mathrm{~mm}$ wide. Pore surface first cream-coloured, later pale ochraceous to dirty orange, even to stepwise-like; 


\begin{tabular}{|c|c|c|c|c|c|}
\hline Species & Distribution & Host trees & Pores per $\mathrm{mm}$ & $\begin{array}{l}\text { Skeletal hyphae } \\
\text { (diameter, arrangement, } \\
\text { reactions if indicative) }\end{array}$ & Basidiospores (shape, size) \\
\hline A. cincta & North America, temperate & Pinus spp. & $6-7$ & $\begin{array}{l}\text { 2.6-3.7 } \mu \mathrm{m} \text {, irregular, } \\
\text { dissolving in } \mathrm{KOH}\end{array}$ & Ellipsoid to thick cylindrical, $3.7-7.2 \times 2.5-3.4 \mu \mathrm{m}$ \\
\hline A. crassa & Eurasia, boreal & Pinus spp. & $6-7$ & $\begin{array}{l}2.3-4.2 \mu \mathrm{m} \text {, irregular, } \\
\text { dissolving in } \mathrm{KOH}\end{array}$ & Ellipsoid to narrowly ellipsoid, $4.9-8.2 \times 3.0-3.8 \mu \mathrm{m}$ \\
\hline A. cretacea & $\begin{array}{l}\text { North America, Eurasia, } \\
\text { boreal - temperate }\end{array}$ & $\begin{array}{l}\text { Various conifers, mostly } \\
\text { Picea spp. }\end{array}$ & $4-6$ & $\begin{array}{l}2.6-4.1 \mu \mathrm{m} \text {, irregular, } \\
\text { dissolving in } \mathrm{KOH}\end{array}$ & Narrowly ellipsoid to thick cylindrical, $4.6-7.9 \times 2.7-4.0 \mu \mathrm{m}$ \\
\hline A. ferox & $\begin{array}{l}\text { North America, Eurasia, } \\
\text { warm temperate - subtropical }\end{array}$ & Juniperus spp. & $4-6$ & $2-3 \mu \mathrm{m}$, subparallel & Fusiform to navicular, $5.6-9.3 \times 2.6-4.0 \mu \mathrm{m}$ \\
\hline A. ignobilis & $\begin{array}{l}\text { North-America, South-West, } \\
\text { warm temperate }\end{array}$ & Pinus spp. & $6-8$ & $2.3-3.6 \mu \mathrm{m}$, irregular & Thick cylindrical, $3.7-5.3 \times 2.0-2.4 \mu \mathrm{m}$ \\
\hline A. ladiana & $\begin{array}{l}\text { North America, warm } \\
\text { temperate - subtropical }\end{array}$ & Pinus spp. & $4-6$ & 2.0-4.1 $\mu \mathrm{m}$, subparallel & Cylindrical, $4.5-6.1 \times 1.6-2.1 \mu \mathrm{m}$ \\
\hline A. piceata & Eurasia, boreal - temperate & $\begin{array}{l}\text { Various conifers, mostly } \\
\text { Picea spp. }\end{array}$ & $5-7$ & $2.5-4.2 \mu \mathrm{m}$, irregular, amyloid & Cylindrical to thick cylindrical, $3.9-6.8 \times 1.7-2.8 \mu \mathrm{m}$ \\
\hline A. pinea & East Asia, temperate - subtropical & Pinus letteri, Picea ajanensis & $6-8$ & 2.0-3.3 $\mu \mathrm{m}$, subparallel & Cylindrical, $5.0-6.1 \times 1.8-2.0 \mu \mathrm{m}$ \\
\hline A. pini-cubensis & $\begin{array}{l}\text { Caribbean, warm } \\
\text { temperate - subtropical }\end{array}$ & Pinus spp. & $6-7$ & 2.1-3.2 $\mu \mathrm{m}$, subparallel & Cylindrical, $5.2-6.2 \times 2.0-2.2 \mu \mathrm{m}$ \\
\hline A. sitchensis & North-America, North-West & $\begin{array}{l}\text { Various conifers, mostly } \\
\text { Picea spp. }\end{array}$ & $4-6$ & 2.3-4.2 $\mu \mathrm{m}$, irregular, amyloid & Cylindrical to thick cylindrical, $4.1-6.8 \times 1.9-2.9 \mu \mathrm{m}$ \\
\hline A. sordida & North-America, North-East & Picea spp. & $6-8$ & 2.1-3.2 $\mu \mathrm{m}$, irregular, amyloid & Cylindrical, $3.7-6.0 \times 1.7-2.2 \mu \mathrm{m}$ \\
\hline
\end{tabular}


pores roundish to angular, on sloping substrates open, (4) 5-7 per $\mathrm{mm}$, dissepiments first even, later minutely serrate. Section: subiculum white, first leathery, in older basidiocarps degenerating in chalky mass, up to $0.2 \mathrm{~mm}$ thick; tubes concolorous with pore surface, cheese-like in fresh condition, woody hard but rather easily cracking in herbarium specimens, annual layers distinct, 2-15 mm thick. Odour pleasant, reminiscent of clove oil; taste slightly bitter or sour.

Hyphal structure dimitic; hyphae with clamps.

Subiculum. Skeletal hyphae dominating, irregularly arranged, with capillary lumen or subsolid, (1.9-) 2.0-3.0 $(-3.4) \mu \mathrm{m}$ in diam. $(\mathrm{n}=20 / 1)$, amyloid (grey in $\mathrm{KOH})$, slightly swelling in $\mathrm{KOH}$. Generative hyphae with thickened walls, some with oil-rich content, 3-4 $\mu \mathrm{m}$ in diam. Resinous matter abundant, present as droplets up to $10 \mu \mathrm{m}$ wide.

Tubes. Skeletal hyphae dominating, irregularly arranged, thick-walled to subsolid, (2.4-) 2.5-4.2 (-4.3) $\mu \mathrm{m}$ in diam. ( $n=150 / 7)$, amyloid (grey in IKI), slightly swelling in $\mathrm{KOH}$; generative hyphae thin-to slightly thick-walled, 2-3.5 $\mu \mathrm{m}$ in diam. Subhymenium distinct in some specimens, up to $20 \mu \mathrm{m}$ thick. Cystidioles abundant, bullet-shaped, with blunt tips, or sharp-pointed, (10.2-) 10.3-17.6 (-19.3) × (3.6-) 3.7-6.3 (-6.6) $\mu \mathrm{m}(\mathrm{n}=60 / 7)$. Basidia broadly clavate, with narrowed base, (10.2-) 10.3-18.6 (-20.1) × (4.3-) 4.4-7.2 (-7.8) $\mu \mathrm{m}$ ( $n=60 / 5)$. Resinous matter present as small droplets, abundant to scanty.

Basidiospores cylindrical to thick cylindrical (bean-shaped), (3.7-) 3.9-6.8 (-7.4) × (1.6-) 1.7-2.8 $\mu \mathrm{m}(\mathrm{n}=330 / 11), \mathrm{L}=4.63$, $\mathrm{W}=2.10, \mathrm{Q}=1.86-2.49$, ventral side flat or slightly concave, longest spores slightly tapering to the distal end.

Discussion. The distinction of A. piceata from morphologically similar species relies mainly on the DNA characters and distribution ranges. Its closest relatives are A. sitchensis and A. sordida from North America. In earlier treatments of European polypores, A. piceata has been mislabelled with both of these names (David \& Tortić 1984; Niemelä et al. 1992; Vampola \& Pouzar 1992; Ryvarden \& Gilbertson 1993; Ryvarden \& Melo 2014). However, our data show that A. sitchensis and Antrodia sordida do not occur in Eurasia. Morphological differences between these three species are minute. Antrodia sordida seems to be more distinct in morphological terms, having smaller pores, narrower skeletal hyphae, shorter basidia, and slightly narrower basidiospores. In microscopic characters, A. piceata is almost identical to A. sitchensis, and with extremely large infraspecific variation of basidiospores (Table 2). Antrodia piceata has on the average smaller pores than A. sitchensis, and the pore surface of older basidiocarps is more deeply coloured (especially in the winter) and often stepwise.

Distribution and ecology. Most records of A. piceata are from old-growth forests, where it inhabits large lying trunks of conifer trees, mostly Picea spp. It is an Eurasian species, with records from France, Germany, Poland, Czech Republic, Slovakia, Croatia, Estonia, Finland, European part of Russia (Russian Karelia, Leningrad, and Nizhny Novgorod Regions), Russian Far East (Khabarovsk Reg. and Primorie), northern parts of China (Heilongiang) and Japan (Hokkaido), although it is very rare throughout its distribution range.

Specimens examined. China. Heilongiiang: Yichun, Fenglin Nat. Res., Picea sp., 5.VIII.2006 Penttilä 13209 (H). Croatia. Lika-
Senj: Plitvička Jezera Nat. Park, Abies alba, 2.V.1975 Tortić (H). Czech Republic. South Bohemia Reg.: Boubín Nat. Res., P. abies, 29.X.2011 Vlasák 1110/14* (H - holotype, JV - isotype). Estonia. Ida-Virumaa: Muraka, P. abies, 21.X.2005 Lõhmus 595 (TU 121006), Lõhmus 610* (TU 121007), Lõhmus 626* (TU 121008). Jõgevamaa: Torma P. abies 28.IX.2014 Runnel 1094* (TU). Tartumaa: Järvselja P. abies 19.IX.2009 Lõhmus 1989* (TU 121009). Võrumaa: Haanja, P. abies, 26.VII.2000 Parmasto* (TAAM 174833, H). Finland. Etelä-Häme: Padasjoki, Vesijako, P. abies, 15.IX.1999 Miettinen 1229* (H 6012565), Ruovesi, Nuijakorpi, P. abies, 25.VIII.2002 Niemelä 7300 (H). France. Haute-Corse: Ariola, Pinus nigra var. laricio, 20.X.1997 Norstedt 97215* (H ex O 15982). Japan. Hokkaido: Akan, conifer, 23.IX.1994 Núñez 630 (H ex O). Poland. Podlesie Reg.: Hajnówka, Białowieża Nat. Park, P. abies, 30.IV.1960 Domański 797 (TAAM 099957), 2.IX.2007, 2.X.2010 Niemelä 8373, 8732 (H). Russia. Karelia: Kondopoga Dist., Kivach Nat. Res., P. abies, 28.V.1997 Miettinen 141 (H). Khabarovsk Reg.: Khabarovsk Dist., Malyi Niran, P. koraiensis, 6.VIII.2012 Spirin 4957 (H); Solnechnyi Dist., Igdomi, P. ajanensis, 6.VIII.2011 Spirin 3885* (H), Razlivnaya, P. ajanensis, 22-24.VIII.2011 Spirin 4249*, 4384* (H). Leningrad Reg.: Podporozhie Dist.: Tokari, P. abies, 27.IX.2007 Spirin 2729 (H), Vazhino, P. abies, 29.IX.2010 Spirin 3567, 3569, 3570 (H). Nizhny Novgorod Reg.: Vetluga Dist., Klenovik Nat. Res., A. sibirica, 9.VIII.1999 Spirin 1401 (H). Primorie Reg.: Chuguevo Dist., Bulyga-Fadeevo, P. ajanensis, 6.IX.1975 Parmasto (TAAM 59287, H). Slovakia. Banska Bystrica Reg.: Čierný Balog, Dobročský Prales, P. abies, 26.VIII.1986 Pouzar (H ex PRM 870295).

Antrodia pinea (B.K. Cui \& Y.C. Dai) Spirin, comb. nov. Fig 5

$\equiv$ Amyloporia pinea B.K. Cui \& Y.C. Dai, Antonie van Leeuwenhoek J. Microb. 104: 821, 2013.

MB 813075

Holotype. China. Hainan: Changjiang Co., Bawangling Nat. Res., Pinus latteri, 10.V.2009 Cui 6522 (BJFC, H - isotype).

Basidiocarps resupinate, effused (up to $20 \mathrm{~cm}$ in widest dimension), perennial. Margin first narrow (up to $0.3 \mathrm{~mm}$ wide), pale cream-coloured, in older basidiocarps degenerating. Pore surface pale yellow to pale ochraceous, in older basidiocarps with grey hues; pores roundish to angular, on sloping surfaces elongated, 6-8 per $\mathrm{mm}$, dissepiments thin, opaque, even or slightly wavy. Section: subiculum first white, thin (up to $0.2 \mathrm{~mm}$ ), later degenerating in crumbling amorphous mass; tubes one-layered or indistinctly stratified, soft chalky, easily crumbling, 0.5-1.5 mm thick. No distinct odour; taste mild.

Hyphal structure dimitic; hyphae with clamps.

Subiculum. Skeletal hyphae dominating, irregularly arranged, with capillary lumen or subsolid, (1.9-) 2.0-2.7 $(-2.8) \mu \mathrm{m}$ in diam. $(\mathrm{n}=20 / 1)$, inamyloid, slightly swelling in $\mathrm{KOH}$. Generative hyphae rare, with thickened walls, 1.5-2.5 $\mu \mathrm{m}$ in diam. Resinous matter present in senescent parts as irregular bodies on hyphae.

Tubes. Skeletal hyphae dominating, subparallel, thickwalled with capillary lumen, (1.8-) 2.0-3.3 (-3.4) $\mu \mathrm{m}$ in diam. ( $n=40 / 2)$, inamyloid, slightly swelling in $\mathrm{KOH}$; generative hyphae thin-to slightly thick-walled, $2-3 \mu \mathrm{m}$ in diam. Subhymenium distinct in some parts, up to $30 \mu \mathrm{m}$ thick. Cystidioles abundant, bottle-shaped with sharp tips, rarely 
tapering blunt-tipped, (9.8-) 9.9-13.4 (-15.0) × (3.2-) 3.5-4.9 $(-5.3) \mu \mathrm{m}(\mathrm{n}=20 / 1)$. Basidia clavate, with narrowed base, (9.7-) 10.1-14.7 (-15.8) × (4.4-) 4.6-5.3 (-5.4) $\mu \mathrm{m}(\mathrm{n}=20 / 1)$. Resinous matter abundant, present in older basidiocarps as globules or large amorphous bodies up to $30 \mu \mathrm{m}$ wide. Rhomboid crystals abundant on tramal hyphae, up to $25 \mu \mathrm{m}$ in longest dimension.

Basidiospores cylindrical, (4.7-) 5.0-6.1 (-6.2) $\times(1.7-)$ 1.8-2.0 (-2.2) $\mu \mathrm{m}(\mathrm{n}=30 / 1), \mathrm{L}=5.48, \mathrm{~W}=1.92, \mathrm{Q}=2.87$, ventral side flat, longest spores arcuate near apiculus and slightly tapering to the distal end.

Discussion. This species was recently described as belonging to the genus Amyloporia (Cui \& Dai 2013) as outlined by Rajchenberg et al. (2011). Morphologically, it is most similar to the North American A. ladiana and A. pini-cubensis. Antrodia piceata, widely distributed in the boreal zone of Eurasia, differs from A. pinea in having larger and tougher basidiocarps, wider pores and amyloid skeletal hyphae.

Distribution and ecology. Antrodia pinea was described based on several collections from the southern part of China (Cui \& Dai 2013), and it was reported as growing on lying trunks of Pinus spp. We found it also from Russian Far East on a lying trunk of P. ajanensis. The species evidently prefers xerophilic habitats.

Specimens examined. China. Hainan: Changjiang Co., Bawangling Nat. Res., Pinus latteri, 10.V.2009 Cui 6522* (BJFC holotype, $\mathrm{H}$ - isotype). Russia. Khabarovsk Reg.: Solnechnyi Dist., Suluk-Makit, P. ajanensis, 19.VIII.2011 Spirin 4211* (H).

Antrodia pini-cubensis Vampola, Kotlaba \& Pouzar, Czech Mycol. 47 (3): 190, 1994 - Fig 5

Holotype. Cuba. Oriente Prov.: Mayarí, Sierra Nipe, Pinus cubensis, 13.III.1967 Kotlaba (PRM 756464).

Basidiocarps resupinate, effused (up to $7 \mathrm{~cm}$ in widest dimension), perennial. Margin first narrow (up to $0.5 \mathrm{~mm}$ wide), white to pale cream-coloured, in older basidiocarps degenerating. Pore surface bright ochraceous, contrasting with paler margin, in older basidiocarps with resinous-brown hues; pores round, more or less regular, on sloping surfaces elongated, (5) 6-7 per mm, dissepiments rather thick, opaque, even or slightly wavy. Section: subiculum first white, very thin (less than $0.1 \mathrm{~mm}$ ), later degenerating in crumbling amorphous mass; tubes one-layered or indistinctly stratified, soft leathery or chalky, $0.5-2 \mathrm{~mm}$ thick. No distinct odour; taste mild.

Hyphal structure dimitic; hyphae with clamps.

Subiculum. Skeletal hyphae dominating, with capillary lumen or subsolid, (2.0-) 2.2-2.7 $(-2.8) \mu \mathrm{m}$ in diam. $(\mathrm{n}=20 / 1)$, inamyloid, slightly swelling in $\mathrm{KOH}$. Generative hyphae rare, with thickened walls, $1.5-2.5 \mu \mathrm{m}$ in diam. Resinous matter present as irregular bodies on hyphae.

Tubes. Skeletal hyphae dominating, subparallel, thickwalled to subsolid, (1.9-) 2.1-3.2 (-3.3) $\mu \mathrm{m}$ in diam. $(\mathrm{n}=40 /$ 2), inamyloid, slightly swelling in $\mathrm{KOH}$; generative hyphae thin-to slightly thick-walled, $2-3 \mu \mathrm{m}$ in diam. Subhymenium distinct in some parts, up to $10 \mu \mathrm{m}$ thick. Cystidioles abundant, bullet-shaped, with blunt tips, rarely tapering, (8.4-) 9.0-13.2 (-13.8) × (4.1-) 4.2-5.9 (-6.3) $\mu \mathrm{m}(\mathrm{n}=20 / 1)$. Basidia broadly clavate, with narrowed base, (9.2-) 9.7-13.7 $(-13.8) \times(5.2-) 5.3-6.6(-6.7) \mu \mathrm{m}(\mathrm{n}=20 / 1)$. Resinous matter abundant, present as small droplets, in older basidiocarps producing large amorphous bodies up to $20 \mu \mathrm{m}$ wide.

Basidiospores cylindrical, (5.1-) 5.2-6.2 (-6.4) $\times(1.9-)$ 2.0-2.2 (-2.3) $\mu \mathrm{m}(\mathrm{n}=30 / 1), \mathrm{L}=5.48, \mathrm{~W}=2.09, \mathrm{Q}=2.63$, ventral side flat, longest spores slightly tapering to the distal end.

Discussion. The original description of A. pini-cubensis (Vampola et al. 1994) was based on two specimens from the eastern part of Cuba collected on P. cubensis. Its differences from the morphologically similar A. ladiana are listed above.

Distribution and ecology. Antrodia pini-cubensis is distributed in the Caribbean and so far known from Cuba and Florida.

Specimens examined. Cuba. Oriente Prov.: Mayarí, Sierra Nipe, P. cubensis, 13.III.1967 Kotlaba (PRM 756464 - holotype). USA. Florida: Collier Co., Everglades Nat. Park, P. elliottii, 29.VIII.2010 Vlasák 1008/66* (JV, H).

Antrodia sitchensis (D.V. Baxter) Gilb. \& Ryvarden, Mycotaxon 22 (2): 364, 1985 - Figs 4 and 5

$\equiv$ Poria sitchensis D.V. Baxter, Pap. Michigan Acad. Sci. 23: 293, 1938 ('1937').

Holotype. USA. Alaska: Sitka Co., Sitka, Picea sitchensis, 1.IX.1933 Baxter (MICH 11825).

Basidiocarps resupinate, large, covering several decimetres, perennial. Margin distinct, first whitish to cream-coloured, sterile, leathery, firmly attached or partly detaching, in older basidiocarps becoming resinous and dark brown to almost black, up to $2 \mathrm{~mm}$ wide. Pore surface first cream-coloured, later pale ochraceous, even to indistinctly stepwiselike; pores roundish to angular, on sloping substrates open, 4-6 (7) per mm, dissepiments first even, later minutely serrate. Section: subiculum white, first leathery, in older basidiocarps degenerating in chalky mass, up to $0.2 \mathrm{~mm}$ thick; tubes concolorous with pore surface, cheese-like in fresh condition, woody hard but rather easily cracking in herbarium specimens, annual layers distinct, 1.5-12 mm thick. Odour pleasant, sweet; taste slightly bitter or sour.

Hyphal structure dimitic; hyphae with clamps.

Subiculum. Skeletal hyphae dominating, mostly solid, (2.8-) 2.9-4.0 (-4.2) $\mu \mathrm{m}$ in diam. $(\mathrm{n}=20 / 1)$, amyloid (grey in $\mathrm{KOH})$, slightly swelling in $\mathrm{KOH}$. Generative hyphae with thin or thickened walls, $2.5-3.5 \mu \mathrm{m}$ in diam. Resinous matter abundant, present as droplets up to $15 \mu \mathrm{m}$ wide.

Tubes. Skeletal hyphae dominating in trama, irregularly arranged, thick-walled to subsolid, (2.1-) 2.3-4.2 (-4.7) $\mu \mathrm{m}$ in diam. ( $\mathrm{n}=120 / 6)$, amyloid (grey in IKI), slightly swelling in $\mathrm{KOH}$; generative hyphae thin-to slightly thick-walled, $2.5-4 \mu \mathrm{m}$ in diam. Subhymenium distinct in some specimens, up to $20 \mu \mathrm{m}$ thick. Cystidioles abundant, bullet-shaped, with blunt tips, or sharp-pointed, (9.8-) 10.8-19.2 (-19.4) $\times(3.4-)$ 3.7-5.3 $(-5.5) \mu \mathrm{m}(\mathrm{n}=40 / 4)$. Basidia broadly clavate, with narrowed base, (12.0-) 13.6-20.0 (-20.2) × (4.7-) 5.1-7.1 (-7.2) $\mu \mathrm{m}$ $(n=50 / 3)$. Resinous matter present as small droplets, abundant to scanty.

Basidiospores cylindrical to thick cylindrical (bean-shaped), (3.9-) 4.1-6.8 (-7.6) × (1.8-) 1.9-2.9 (-3.2) $\mu \mathrm{m}(\mathrm{n}=180 / 6)$, $\mathrm{L}=4.80, \mathrm{~W}=2.21, \mathrm{Q}=1.79-2.48$, ventral side flat or slightly concave, longest spores slightly tapering to the distal end.

Discussion. This species was described by Baxter (1938) from the Pacific coast of Alaska. We studied its type as well as all 
available specimens and newly obtained collections, some of which were sequenced. Our results show that most of the specimens labelled as A. sitchensis represent two distinct species and this name may be applied only to the species distributed in the American North-West. The closely related A. piceata and A. sordida grow in Eurasia and American NorthEast, respectively.

Distribution and ecology. Antrodia sitchensis is a temperate species that is known from virgin or near-virgin conifer forests. It grows on lying trunks of several coniferous tree genera (Larix, Pinus, Picea, Pseudotsuga) but seems to be rare throughout its geographic range that stretches from Alaska to the western part of Canada (British Columbia) and the US NorthWest (Montana, Oregon, Washington, northern part of California). Reports of this species from Arizona (Gilbertson 1974) and New York (Lowe 1966; Gilbertson \& Ryverden 1986) represent A. ignobilis and A. sordida, respectively. Records of A. sitchensis from Eurasia belong to A. cretacea and A. piceata.

Specimens examined. Canada. British Columbia: Natural Bridge, Pinus contorta, 3.VIII.1960 Hughes (H, O ex DAOM 125959); Queen Charlotte Ids., Curch Creek, Picea sitchensis, 10.VII.1946 Forster (H ex DAOM 125027). USA. Alaska: Sitka Co., Sitka, P. sitchensis, 1.IX.1933 Baxter (MICH 11825 - holotype); Thorne Bay, P. sitchensis, 13.VII.1954 Kimmey (NY). California: Humboldt Co., Fern Canyon, P. sitchensis, IX.2007 Vlasák 0709/ $77^{*}(\mathrm{~V}, \mathrm{H})$. Montana: Glacier Co., Glacier Nat. Park, Larix occidentalis, 2.VIII.1952 Gilbertson 324 (H ex O). Oregon: Clatsop Co., Astoria, Pseudotsuga menziesii, 8.VII.1960 Davidson* (CFMR FP-105587). Washington: Jefferson Co., Hoh River, P. sitchensis, 19.VIII.1957 Lowe 7834* (NY), 20.X.2014 Spirin 8782* (H); Pend Oreille Co., Slate Creek, L. occidentalis, 15.X.2014 Miettinen 18825 (H).

Antrodia sordida Ryvarden \& Gilb., Mycotaxon 19: 143, 1984 - Figs 4 and 5

Holotype. USA. New Hampshire: Carroll Co., North Conway, Picea rubens, 10.VIII.1920 Snell 557 (BPI 000632).

Basidiocarps resupinate, large, up to $20 \mathrm{~cm}$ in widest dimension, perennial. Margin distinct, first whitish to creamcoloured or pale ochraceous, sterile, leathery, thinning-out firmly attached, in older basidiocarps disappearing, up to $0.5 \mathrm{~mm}$ wide. Pore surface first cream-coloured, later pale ochraceous, in older parts with brownish stains, even; pores roundish to angular, on sloping substrates open, 6-8 per $\mathrm{mm}$, dissepiments first even, later minutely serrate. Section: subiculum white, leathery, up to $0.1 \mathrm{~mm}$ thick; tubes concolorous with pore surface, cheese-like in fresh condition, woody hard but rather easily cracking in herbarium specimens, annual layers distinct, $1-5 \mathrm{~mm}$ thick. Odour faint, pleasant, sweet; taste slightly bitter or sour.

Hyphal structure dimitic; hyphae with clamps.

Subiculum. Skeletal hyphae dominating, with capillary lumen or subsolid, (1.9-) 2.3-3.7 (-4.6) $\mu \mathrm{m}$ in diam. $(\mathrm{n}=20 / 1)$, amyloid (grey in IKI), slightly swelling in $\mathrm{KOH}$. Generative hyphae with thin or thickened walls, sometimes with oil-rich content, 2-3.5 $\mu \mathrm{m}$ in diam. Resinous matter abundant, present as droplets up to $15 \mu \mathrm{m}$ wide.

Tubes. Skeletal hyphae dominating in trama, irregularly arranged, thick-walled to subsolid, (2.0-) 2.1-3.2 (-3.5) $\mu \mathrm{m}$ in diam. ( $\mathrm{n}=80 / 4)$, amyloid (grey in IKI), slightly swelling in
$\mathrm{KOH}$; generative hyphae thin-to slightly thick-walled, $2-3 \mu \mathrm{m}$ in diam. Subhymenium distinct in some specimens, up to $10 \mu \mathrm{m}$ thick. Cystidioles abundant, mostly sharppointed, rarely bullet-shaped, (8.3-) 9.2-15.7 (-16.0) × (3.1-) 3.3-4.5 $(-5.6) \mu \mathrm{m}(\mathrm{n}=20 / 2)$. Basidia broadly clavate, with narrowed base, $(9.3-)$ 10.1-14.8 (-16.6) × (3.8-) 4.3-6.1 (-6.2) $\mu \mathrm{m}$ $(\mathrm{n}=40 / 2)$. Resinous matter present as small droplets, abundant to scanty.

Basidiospores cylindrical, (3.6-) 3.7-6.0 (-6.2) × (1.6-) 1.7-2.2 (-2.3) $\mu \mathrm{m}(\mathrm{n}=120 / 4), \mathrm{L}=4.54, \mathrm{~W}=1.85$, $\mathrm{Q}=2.07-2.59$, ventral side flat or slightly concave.

Discussion. Antrodia sordida was introduced by Overholts (1942) under the name P. oleagina. In the original description, the Latin protologue was omitted, and therefore Ryvarden \& Gilbertson (1984) redescribed it as A. sordida. However, identity of this species remained vague until now. Its differences from the closely related species are listed under A. piceata. Vampola \& Pouzar (1992) checked the type of A. sordida and stated that its skeletal hyphae dissolve in $\mathrm{KOH}$ solution. This is true for the type collection which is now about $100 \mathrm{y}$ old, as well as for many other polypore specimens from various genera, that have been kept for decades. We studied two recent, fully developed and typical collections of A. sordida in which the skeletal hyphae swell in $\mathrm{KOH}$ solution only slightly, in the same manner as in A. sitchensis and A. piceata.

Distribution and ecology. Antrodia sordida grows on lying trunks of Picea spp. in the American North-East. The earlier collection of A. sordida (as P. oleagina) from Minnesota (Gilbertson \& Lombard 1976) belongs to A. cretacea.

Specimens examined. USA. New Hampshire: Carroll Co., North Conway, P. rubens, 10.VIII.1920 Snell 557 (BPI 000632 holotype). New York: Hamilton Co., Adirondack Nat. Park, Fish Creek Point, Picea sp., IX.2005 Vlasák 0509/190* (JV, H), Piseco, Picea sp., 28.VIII.1902 Atkinson (NY), Seventh Lake, conifer, VII.1916 Atkinson (NY); Essex Co., Arbutus Lake, Picea sp., 17.IX.2013 Miettinen 16954* $(\mathrm{H})$.

\section{Discussion}

Morphological and phylogenetic revision of the Antrodia crassa group revealed eleven species (including five new species) in the boreal to subtropical zones of the Northern Hemisphere. The monophyletic group of these species appeared most closely related to species of Antrodia and Amyloporia, including the type of the latter genus, Amyloporia xantha. Similar results from earlier works, comprising only a few members of the A. crassa group, have led to their inclusion in Amyloporia (Rajchenberg et al. 2011; Cui \& Dai 2013). However, recent phylogenetic analyses by Ortiz-Santana et al. (2013) do not support the monophyly of Amyloporia with respect to its expanded concept, introduced by Rajchenberg et al. (2011). Our results distinguished several well-supported groups involving species combined into Amyloporia. However, the larger clade including all of these groups received controversial support and comprised also the type of Rhodonia, Rhodonia placenta. Since the morphological characterisation of the resulting heterogenous assemblage is infeasible, we anticipate further generic splitting of Amyloporia sensu Rajchenberg et al. (2011). For the time being, we prefer to retain the wide concept of Antrodia 
as defined by Ryvarden (1991). Therefore we have described all members of the A. crassa group as species of Antrodia while acknowledging their distant relationship to the type species of the latter genus, Antrodia serpens.

Morphological data also support the distinction of the A. crassa group from the genetically differentiated groups including Amyloporia xantha and Antrodia sinuosa. Amyloporia xantha, and its closest relatives, Antrodia alpina and Antrodia subxantha (Dai et al. 2012; Cui \& Dai 2013), develop a densely packed hymenium consisting of narrow and rather short, thin-walled basidia, and slender, poorly differentiated cystidioles. In addition, basidiospores of these species are allantoid and distinctly curved, and skeletal hyphae in tube trama are strongly amyloid, parallel, with a broad lumen, neither twisted nor inflated. No resinous matter is detected among hyphal tissues. In turn, A. sinuosa produces wide-pored greyish basidiocarps, often with strong smell of licorice, and its skeletal hyphae are parallel, but they are subsolid, inamyloid, brownish, and often bearing large rhomboid crystals. Basidia of A. sinuosa are narrowly clavate, and the basidiospores are clearly curved and narrow, allantoid. By contrast, members of the A. crassa group have tortuous and variably inflated, in most species irregularly arranged skeletal hyphae with capillary or almost invisible lumen; abundant resinous matter occurs in the context and tubes. Moreover, skeletals are swelling and in some species almost totally dissolving in $\mathrm{KOH}$ solution. Basidia of A. crassa and its siblings are loosely arranged, broadly clavate to barrel-shaped, with thickened walls after spore detachment, and basidiospores are cylindrical to broadly ellipsoid, not curved.

The species of the A. crassa group differ from each other by combinations of host tree preference and distribution range. All but one of the six host tree-specific species, Antrodia cincta, A. crassa, A. ignobilis, A. ladiana, and A. pini-cubensis, grow on dry fallen trunks of Pinus spp. Only Antrodia ferox has been found on dead Juniperus trunks. Most of the pine-dwelling species are restricted to a particular vegetation zone in either boreal Eurasia (A. crassa) or subtropical North America (A. ignobilis, A. ladiana, A. pini-cubensis). Antrodia cincta, however, is found both in the subtropical and temperate regions of North-America. The more widely distributed species, A. cretacea, A. piceata, and A. sitchensis, have been found from at least two different genera of conifers, with a preference for Picea spp. While Antrodia cretacea is a holarctic species, Antrodia piceata is known from boreal and temperate Eurasia, and Antrodia sitchensis from the American North-West. The data on Antrodia pinea and Antrodia sordida are insufficient for drawing conclusions on their ecology and distribution.

The habitat data generally indicate that most species of the Antrodia crassa group are potentially threatened by forest management. Among better-known species, both those inhabiting mid-to late successional forests (A. piceata, A. crassa) or post-disturbance habitats (A. cretacea) appear to be restricted to dead-wood rich areas (Junninen 2009; Runnel et al. 2014). The average coarse dead-wood in A. crassa habitats in Finland is $39 \mathrm{~m}^{3} \mathrm{ha}^{-1}$ (Junninen 2009) and in the Estonian clear-cuts hosting A. cretacea $12-41 \mathrm{~m}^{3} \mathrm{ha}^{-1}$ (data extracted from Lõhmus et al. 2013). These amounts exceed the usual dead-wood left by intensive forestry (e.g. Siitonen 2001), while approximating the threshold levels suggested for red-listed polypores in boreal forests (around $20 \mathrm{~m}^{3} \mathrm{ha}^{-1}$; Junninen \& Komonen 2011). Given that, the conspicuous species of the A. crassa group might serve as indicators of the potential occurrence of other dead-wood dependent organisms or of sustainable conservation management of coniferous forests. However, the published lists of threatened and indicator species including the members of this group (e.g. Niemelä 2005; Brandrud et al. 2010; Kotiranta et al. 2010) first require a taxonomic revision.

Several species not mentioned so far may belong to the A. crassa group. Polyporus caseicarnis Speg., described from Argentina, has been placed into the synonyms of A. crassa by Rajchenberg \& Wright (1987); however, the latter authors noted some minor differences from the European material. Ryvarden \& Johansen (1980) mentioned A. crassa and A. sordida (as Antrodiella oleagina) from the mountain areas of East Africa; their identity deserves further study. The records of A. crassa from China (Dai 2000) seem to represent A. cretacea but warrant further study.

\section{Acknowledgements}

The curators of herbaria BJFC, BPI, CFMR, H, MICH, NY, O, PRM, TAAM, TU are thanked for providing specimens for loan. Asko Lõhmus and two anonymous reviewers constructively commented on the manuscript. Rasmus Puusepp did most of the DNA-work. Kare Liimatainen (University of Helsinki) sequenced two older collections of A. sitchensis. Sten Svantesson provided the Swedish specimen of A. cretacea, and Yu-Cheng Dai the type material of A. pinea. The Turkish specimen of A. ferox was provided by Leif Ryvarden. Steve Trudell, Lee Whitford, Andrew and Cathy Parker helped with field trips of the authors VS, OM and KP in the US North-West. Pekka Helo kindly provided us a photograph of A. crassa. The research was supported by the Chancellor's travel grant from the University of Helsinki (VS) and Estonian Research Agency projects ETF9051, IUT34-7 (KR) and IUT20-30 (KP), as well as EU Marie Curie fellowship PIOF-GA-2011-302349 (OM). Molecular work was financed by European Union through the European Regional Development Fund (Centre of Excellence FIBIR at the University of Tartu) and by Czech Republic institutional support RVO:60077344.

\section{Appendix A. Supplementary data}

Supplementary data related to this article can be found at http://dx.doi.org/10.1016/j.funbio.2015.09.008.

\section{R E F E R E N C E S}

Baxter DV, 1938. Some resupinate polypores from the region of the Great Lakes. 9. Papers of the Michigan Academy of Science, Arts and Letters 23: 285-305.

Bondartsev AS, 1953. Trytouye griby Europeiskoi chastii SSSR i Kaukaza. Akademiya Nauk SSSR, Moscow, Leningrad.

Brandrud TE, Bendiksen E, Hellik Hofton T, Høiland K, Jordal JB, 2010. Fungi. In: Kålås JA, Viken $\AA$, Henriksen S, Skjelseth S 
(eds), The 2010 Norwegian Red List for Species. Norwegian Biodiversity Information Centre, Norway, pp. 87-124.

Castresana J, 2000. Selection of conserved blocks from multiple alignments for their use in phylogenetic analysis. Molecular Biology Evolution 17: 540-552.

Cui BK, Dai YC, 2013. Molecular phylogeny and morphology reveal a new species of Amyloporia (Basidiomycota) from China. Antonie van Leeuwenhoek Journal of Microbiology 104: 817-827.

Dai YC, 2000. A checklist of polypores from Northeast China. Karstenia 40: 23-29.

Dai YC, He XS, Wanghe KY, Steffen K, 2012. Wood-decaying fungi in eastern Himalayas 2. Species from Qingcheng Mts., Sichuan Province. Mycosystema 31: 168-173.

David A, Tortić M, 1984. Amyloporiella gen. nov. (Polyporaceae). Transactions of the British Mycological Society 83: 659-667.

Donk MA, 1960. The generic names proposed for Polyporaceae. Persoonia 1: 173-302.

Donk MA, 1966. Notes on European polypores 1. Persoonia 4: 337-343.

Fries EM, 1874. Hymenomycetes Europaei. Berling, Uppsala.

Gardes M, Bruns TD, 1993. ITS primers with enhanced specificity for basidiomycetes e application to the identification of mycorrhizae and rusts. Molecular Ecology 2: 113-118.

Gilbertson RL, 1974. Fungi that Decay Ponderosa Pine. University of Arizona, Tucson.

Gilbertson RL, Lombard FF, 1976. Wood-rotting Basidiomycetes. Itasca State Park annotated list. Journal of the Minnesota Academy of Science 42: 25-31.

Gilbertson RL, Ryvarden L, 1986. North American polypores. 1. Abortiporus to Lindtneria. Fungiflora, Oslo.

Junninen K, 2009. Kalkkikäävän (Antrodia crassa) suojeluselvitys. Metsähallituksen luonnonsuojelujulkaisuja. Sarja A 182: 1-52.

Junninen K, Komonen A, 2011. Conservation ecology of boreal polypores: a review. Biological Conservation 144: 11-20.

Karsten PA, 1879. Symbolae ad mycologiam fennicam 6. Medd. Soc. pro Fauna Flora Fennica 5: 15-46.

Karsten PA, 1889. Kritisk öfvesigt af Finlands Basidsvampar (Basidiomycetes; Gastero- \& Hymenomycetes). Bidrag till Kännedom af Finlands Natur och Folk 48: 1-470.

Katoh K, Toh H, 2008. Recent developments in the MAFFT multiple sequence alignment program. Briefings in Bioinformatics 9: 286-298.

Kotiranta H, Junninen K, Saarenoksa R, Kinnunen J, Kytövuori I, 2010. Aphyllophorales and Heterobasidiomycetes. In: Rassi P, Hyvärinen E, Juslén A, Mannerkoski I (eds), The 2010 Red List of Finnish Species. Ministry of the Environment. Finnish Environment Institute, Helsinki, pp. 249-263.

Kirk PM, Cannon PF, Minter DW, Stalpers JA, 2008. Ainsworth \& Bisby's Dictionary of the Fungi, 10th edn. CAB International, Wallingford, Oxon.

Lanfear R, Calcott B, Ho SYW, Guindon S, 2012. PartitionFinder: combined Selection of Partitioning Schemes and Substitution Models for Phylogenetic Analyses. Molecular Biology and Evolution 29: 1695-1701.

Lanfear R, Calcott B, Kainer D, Mayer C, Stamatakis A, 2014. Selecting optimal partitioning schemes for phylogenomic datasets. BMC Evolutionary Biology 14: 82.

Lõhmus A, Kraut A, Rosenvald R, 2013. Dead wood in clearcuts of semi-natural forests in estonia: site-type variation, degradation, and the influences of tree retention and slash harvest. European Journal of Forest Research 132: 335-349.

Lowe JL, 1956. Type studies of polypores described by Karsten. Mycologia 48: 99-125.

Lowe JL, 1966. Polyporaceae of North America. The genus Poria. State University College of Forestry at Syracuse University Technical Publication 90: 1-183.

Matheny PB, Wang Z, Binder M, Curtis JM, Lim YW, Nilsson RH, Hughes KW, Hofstetter V, Ammirati JF, Schoch CL, Langer E,
Langer G, McLaughlin DJ, Wilson AW, Froslev T, Ge ZW, Kerrigan RW, Slot JC, Yang ZL, Baroni TJ, Fischer M, Hosaka K, Matsuura K, Seidl MT, Vauras J, Hibbett DS, 2007. Contributions of rpb2 and tef1 to the phylogeny of mushrooms and allies (Basidiomycota, Fungi). Molecular Phylogenetics and Evolution 43: 430-451. http://dx.doi.org/10.1016/j.ympev.2006.08.024.

Miettinen O, Niemelä T, Spirin W, 2006. Northern Antrodiella species: the identity of A. semisupina, and type studies of related taxa. Mycotaxon 96: 211-239.

Miettinen O, Spirin V, Niemelä T, 2012. Notes on the genus Aporpium (Auriculariales, Basidiomycota), with a new species from temperate Europe. Annales Botanici Fennici 49: 359-368.

Miller MA, Pfeiffer W, Schwartz T, 2010. Creating the CIPRES Science Gateway for inference of large phylogenetic trees. In: Proceedings of the Gateway Computing Environments Workshop (GCE), 14 Nov. 2010, New Orleans, LA, pp. 1-8.

Niemelä T, 2005. Käävät, puiden sienet. Norrlinia 13: 1-320.

Niemelä T, Kotiranta H, Penttilä R, 1992. New records of rare and threatened polypores in Finland. Karstenia 32: 81-94.

Niemelä T, Wallenius T, Kotiranta H, 2002. The kelo tree, a vanishing substrate of specified wood-inhabiting fungi. Polish Botanical Journal 47: 91-101.

Niskanen T, Kytövuori I, Liimatainen K, 2009. Cortinarius sect. brunnei (Basidiomycota, Agaricales) in North Europe. Mycological Research 113: 182-206.

Núñez M, Ryvarden L, 2001. East Asian polypores. Vol. 2. Polyporaceae s. lato. Synopsis Fungorum 14 169-522.

Ortiz-Santana B, Linder DL, Miettinen O, Justo A, Hibbett DS, 2013. A phylogenetic overwiew of the antrodia clade. Mycologia 105: 1391-1411.

Overholts LO, 1942. Polyporaceae of Pennsylvania. The Pennsylvania State College Agricultural Experimental Station Technical Bulletin 418: 1-64.

Pilát A, 1936-1942. Polyporaceae I, II. In: Kavina K, Pilát A (eds), Atlas des Champignons de l'Europe. Vol. 3. Praha (privately published) (part I, text; part II, photographs).

Rajchenberg M, Gorjón SP, Pildain MB, 2011. The phylogenetic disposition of Antrodia s.l. taxa (Polyporales, Basidiomycota) from Patagonia, Argentina. Australian Systematic Botany 24: 111-120.

Rajchenberg M, Wright J, 1987. Type studies of Corticiaceae and Polyporaceae (Aphyllophorales) described by C. Spegazzini. Mycologia 79: 246-264.

Romell L, 1926. Remarks on some species of Polyporus. Suensk Botanisk Tidskrift 20: 1-24.

Ronquist F, Teslenko M, van der Mark P, Ayres DL, Darling A, Höhna S, Larget B, Liu L, Suchard MA, Huelsenbeck JP, 2012. MrBayes 3.2: efficient Bayesian phylogenetic inference and model choice across a large model space. Systematic Biology 61 539-542.

Rostkovius FW, 1848. Die Pilze Deutschlands. In: Sturm I (ed.), Deutschlands Flora in Abbildungen nach Natur mit Beschreibungen, III Abteil. H. 27-28, pp. 1-48.

Runnel K, Põldmaa K, Lõhmus A, 2014. 'Old-forest fungi' are not always what they seem: the case of Antrodia crassa. Fungal Ecology 9: 27-33.

Ryvarden L, 1973. Some genera of resupinate polypores, with a note on Aleurodiscus norvegicus nov. sp. Norwegian Journal of Botany 20: 7-11.

Ryvarden L, 1991. Genera of polypores. Synopsis Fungorum 5: $1-363$.

Ryvarden L, Gilbertson RL, 1984. Type studies in the Polyporaceae 15. Species described by L.O. Overholts, either alone or with J.L. Lowe. Mycotaxon 19: 137-144.

Ryvarden L, Gilbertson RL, 1993. European polypores. 1. Synopsis Fungorum 6: 1-387. 
Ryvarden L, Johansen I, 1980. A Preliminary Polypore Flora of East Africa. Fungiflora, Oslo.

Ryvarden L, Melo I, 2014. Poroid fungi of Europe. Synopsis Fungorum 31: 1-455.

Siitonen J, 2001. Forest management, coarse woody debris and saproxylic organisms: fennoscandian boreal forests as an example. Ecological Bulletins 49: 11-41.

Spirin V, Vlasák J, Niemelä T, Miettinen O, 2013. What is Antrodia sensu stricto? Mycologia 105: 1555-1576.

Stamatakis A, 2014. RA $\times$ ML version 8: a tool for phylogenetic analysis and post-analysis of large phylogenies. Bioinformatics 30: 1312-1313.

Tamm H, Põldmaa K, 2013. Diversity, host associations and phylogeography of temperate aurofusarin-producing Hypomyces/Cladobotryum including causal agents of cobweb disease of cultivated mushrooms. Fungal Biology 117: 348-367.

Thiers B, 2015. Index Herbariorum: a global directory of public herbaria and associated stuff [continuously updated]. New York Botanical Garden's Virtual Herbarium. http://sweetgum.nybg.org/ih [accessed 2 February 2015].
Tedersoo L, Jairus T, Horton BM, Abarenkov A, Suvi T, Saar I, Kõljalg U, 2008. Strong host preference of ectomycorrhizal fungi in a Tasmanian wet sclerophyll forest as revealed by DNA barcoding and taxon-specific primers. New Phytologist 180: 479-490. http://dx.doi.org/10.1111/j.14698137.2008.02561.x.

Trass H, Vellak K, Ingerpuu N, 1999. Floristical and ecological properties for identifying of primeval forests in Estonia. Annales Botanici Fennici 36: 67-80.

Vampola P, Pouzar Z, 1992. Contribution to the knowledge of a rare resupinate polypore Amyloporia sitchensis. Česká Mykologie 46: 213-222.

Vampola P, Kotlaba F, Pouzar Z, 1994. Antrodia pini-cubensis, a new polypore from the Caribbean area. Czech Mycology 47: 189-192.

White TJ, Bruns TD, Lee S, Taylor JW, 1990. Amplification and direct sequencing of fungal ribosomal RNA genes for phylogenetics. In: Innis MA, Gelfand H, Sninsky JS, White TJ (eds), PCR Protocols: a guide to methods and applications. Academic Press, San Diego, pp. 315-322. 